\title{
Stellar populations in gas-rich galaxy mergers II. Feedback effects of Type Ia and II supernovae
}

\author{
Kenji Bekki \& Yasuhiro Shioya ${ }^{1}$ \\ Astronomical Institute, Tohoku University, Sendai, 980-8578, Japan \\ email address: bekki@astroa.astr.tohoku.ac.jp
}

Received —

\footnotetext{
${ }^{1}$ Center for Interdisciplinary Research, Tohoku University, Sendai, 980-8578, Japan
} 


\begin{abstract}
We numerically investigate chemodynamical evolution of major disk-disk galaxy mergers in order to explore the origin of mass-dependent chemical, photometric, and spectroscopic properties observed in elliptical galaxies. We particularly investigate the dependence of the fundamental properties on merger progenitor disk mass $\left(M_{\mathrm{d}}\right)$. Main results obtained in this study are the following five.

(1) More massive (luminous) ellipticals formed by galaxy mergers between more massive spirals have larger metallicity $(Z)$ and thus show redder colors: The typical metallicity ranges from $\sim 1.0$ solar abundance $(Z \sim 0.02)$ for ellipticals formed by mergers with $M_{\mathrm{d}}=10^{10} M_{\odot}$ to $\sim 2.0$ solar $(Z \sim 0.04)$ for those with $M_{\mathrm{d}}=10^{12} M_{\odot}$.

(2) The absolute magnitude of negative metallicity gradients developed in galaxy mergers is more likely to be larger for massive ellipticals. Absolute magnitude of metallicity gradient correlates with that of age gradient in ellipticals in the sense that an elliptical with steeper negative metallicity gradient is more likely to show steeper age gradient.

(3) Radial color gradient is more likely to be larger for more massive ellipticals, which reflects that the metallicity gradient is larger for more massive ellipticals. For example, typical $U-R$ color gradient $(\Delta U-R / \Delta \log R)$ for $0.1 \leq R / R_{\mathrm{e}} \leq 1.0$ is -0.13 for ellipticals with $M_{\mathrm{d}}=10^{12} M_{\odot}$ and -0.07 for those with $M_{\mathrm{d}}=10^{10} M_{\odot}$.

(4) Both $\mathrm{Mg}_{2}$ line index in the central part of ellipticals $\left(R \leq 0.1 R_{\mathrm{e}}\right)$ and radial gradient of $\mathrm{Mg}_{2}\left(\Delta \mathrm{Mg}_{2} / \Delta \log R\right)$ are more likely to be larger for massive ellipticals. $\Delta \mathrm{Mg}_{2} / \Delta \log R$ correlates reasonably well with the central $\mathrm{Mg}_{2}$ in ellipticals. For most of the present merger models, ellipticals show positive
\end{abstract}


radial gradient of $\mathrm{H}_{\beta}$ line index.

(5) Both $M / L_{B}$ and $M / L_{K}$, where $M, L_{B}$, and $L_{K}$ are total stellar mass of galaxy mergers, $B$-band luminosity, and $K$-band one, respectively, depend on galactic mass in such a way that more massive ellipticals have larger $M / L_{B}$ and smaller $M / L_{K}$.

The essential reason for the derived mass-dependent chemical, photometric, and spectroscopic properties of ellipticals is that galactic mass can largely determine total amount of metal-enriched interstellar gas, star formation histories of galaxy mergers, and the effectiveness of Type Ia and II supernova feedback, all of which greatly affect chemodynamical evolution of galaxy mergers.

Subject headings: galaxies: elliptical and lenticular, cD - galaxies: formation galaxies- interaction - galaxies: structure - galaxies: stellar content - galaxies: abundances 


\section{Introduction}

Empirical physical relations in elliptical galaxies, such as the Fundamental Plane (FP) and the color-magnitude (CM) relation, are generally considered to provide strong constraints on the formation and evolution of elliptical galaxies, such as the formation epoch, the star formation histories, and the merging histories (Faber 1973; Visvanathan \& Sandage 1977; Djorgovski \& Davis 1987; Dressler et al. 1987; Bower, Lucey, \& Ellis 1992; Aragón-Salamanca et al. 1993; Djorgovski, Pahre, \& de Carvalho 1996; Franx \& van Dokkum 1996). Physical origins of these relations accordingly have been extensively explored by observational and theoretical studies from variously different points of views (e.g., Arimoto \& Yoshii 1987; Bender, Burstein, \& Faber 1992; Djorgovski et al. 1996; Kauffmann \& Charlot 1997). Among possibly key determinants for the origins, the nature of stellar populations of ellipticals, such as age and metallicity distribution, is recognized as a primarily important factor, principally because stellar populations are generally considered to affect both the slope and tightness of these empirical relations. Origins of such important nature of stellar populations in elliptical galaxies have been indeed investigated mostly in the context of monolithic collapse model with the so-called galactic wind (Larson 1975; Carlberg 1984; Arimoto \& Yoshii 1987; Bressan et al. 1996, Gibson and Matteucci 1997),

they have not been so extensively explored, however, in the context of the merger scenario that ellipticals are formed by major disk-disk galaxy mergers (e.g., Toomre \& Toomre 1972). Accordingly, it is vital to investigate whether or not the merger scenario, which has been already confirmed to have some advantages in reproducing a number of important structural and kinematical properties of ellipticals (e.g., Barnes \& Hernquist 1992), can also reproduce fundamental properties of stellar populations inferred from empirical relations of elliptical galaxies.

There are only a few studies trying to clarify the origin of stellar populations of 
elliptical galaxies along the merger scenario. Schweizer \& Seitzer (1992) demonstrated that the bluer integrated $U B V$ color of elliptical galaxies with morphologically fine structure can be explained by the secondary starburst induced by major disk-disk galaxy mergers. Fritze -v. Alvensleben \& Gerhard (1994) showed that even late galaxy mergers with their age $\sim 12$ Gyr can develop typical colors of ellipticals within $3 \sim 4$ Gyr after the secondary starburst. Kauffmann \& Charlot (1997) constructed a semi-analytic model of elliptical galaxy formation, which is based upon the hierarchical clustering in CDM universe and includes rather simple chemical enrichment process, and thereby demonstrated that the origin of the color-magnitude relation of elliptical galaxies can be reproduced successfully in the CDM model of galaxy formation. These previous studies, which are based on one-zone chemical evolution models, have completely neglected the importance of dynamical effects on chemophotometric evolution of galaxy mergers. Since star formation history, which is one of the most critical determinants for fundamental properties of stellar populations, is considered to depend largely on galactic dynamics in various ways (e.g., Kennicutt 1989; Larson 1992), we should investigate dynamical effects on stellar populations of galaxies in an admittedly self-consistent manner, in order to understand more deeply and thoroughly the nature of stellar populations of elliptical galaxies.

The purpose of this paper is to investigate how dynamical effects of galaxy merging, in particular, violent relaxation combined with gaseous dissipation, affect global properties of stellar populations (e.g. mean stellar metallicity and age) and their radial gradients. The present study is an extended version of our previous study (Bekki \& Shioya 1998, hereafter referred to as Paper I), which is the first step toward the understanding of a close physical relationship between dynamical evolution and chemical one in high redshift galaxy mergers (the redshift of $z=1 \sim 2$ ) between disk galaxies with the gas mass fraction larger than 0.2 that is a typical value of the present late-type spirals. The importance of investigating high redshift gas-rich galaxy mergers is described in Paper I. The present chemodynamical model 
is more realistic and sophisticated than our previous one in the following three points:

(1) Instead of using instantaneous chemical mixing approximation, we consider time-delay between star formation and metal ejection mainly from Type Ia and II supernovae. (2) Feedback effects of Type Ia and II supernovae on dynamical and chemical evolution of galaxy mergers are incorporated. (3) Time-evolution of enrichment processes is solved for each of chemical components, H, He, Mg, O, and Fe. This chemodynamical model enables us to reveal not only physical origins of global (mean) properties of stellar populations, which are the key determinants for galactic empirical physical relations, but also radial gradients of stellar populations in elliptical galaxies. By using the present chemodynamical model, we particularly investigate the origin of mass-dependent chemical, photometric, and spectroscopic properties observed in elliptical galaxies. The layout of this paper is as follows. In $\S 2$, we summarize numerical models used in the present study and describe methods for analyzing the stellar populations produced by dissipative galaxy mergers with star formation. In $\S 3$, we demonstrate how a number of fundamental characteristics of stellar populations in merger remnants depend on galactic mass. In $\S 4$, we provide a number of implications on the formation and evolution of elliptical galaxies, based on the derived results. The conclusions of the present study are given in $§ 5$.

\section{Model}

Chemical, photometric, and spectroscopic evolution of galaxies are basically determined by age and metallicity distribution of stellar populations within galaxies. Fundamental properties of the distribution are furthermore controlled by star formation histories, which are considered to depend strongly on dynamical and kinematical properties of galaxies such as local dynamical instability (e.g. Kennicutt 1989; Larson 1992). Hence we numerically solve the time evolution of chemical, photometric, and spectroscopic evolution of galaxy 
mergers, based on the dynamical evolution of galaxies. Firstly we describe a numerical model for dynamical evolution of galaxy mergers, including structure and kinematics of merger progenitor disks, initial orbital configurations of galaxy mergers, and the prescriptions of dissipative process (§2.1). Secondly, we describe a star formation model, in which gas consumption rate of star formation and the effectiveness of Type Ia and II supernovae feedback are given in detail (§2.2). Thirdly we give the method for analyzing the chemical enrichment process during dissipative galaxy merging with star formation and the photometric properties of merger remnants (§2.3). Lastly, we describe the main points of analysis of the present study $(\S 2.4)$.

\section{1. $\quad$ Merger model}

\subsubsection{Structure and kinematics of merger progenitor disks}

We construct models of galaxy mergers between gas-rich disk galaxies with equal mass by using Fall-Efstathiou model (1980). The total mass and the size of a progenitor disk are $M_{\mathrm{d}}$ and $R_{\mathrm{d}}$, respectively. From now on, all the mass and length are measured in units of $M_{\mathrm{d}}$ and $R_{\mathrm{d}}$, respectively, unless specified. Velocity and time are measured in units of $v=$ $\left(G M_{\mathrm{d}} / R_{\mathrm{d}}\right)^{1 / 2}$ and $t_{\mathrm{dyn}}=\left(R_{\mathrm{d}}^{3} / G M_{\mathrm{d}}\right)^{1 / 2}$, respectively, where $G$ is the gravitational constant and assumed to be 1.0. Dimensional values for these units in each model are given later. In the present model, the rotation curve becomes nearly flat at $0.35 R_{\mathrm{d}}$ with the maximum rotational velocity $v_{\mathrm{m}}=1.8$. The corresponding total mass $M_{\mathrm{t}}$ and halo mass $M_{\mathrm{h}}$ are 3.8 and 2.8 in our units, respectively. The radial $(R)$ and vertical $(Z)$ density profile of a disk are assumed to be proportional to $\exp \left(-R / R_{0}\right)$ with scale length $R_{0}=0.2$ and to $\operatorname{sech}^{2}\left(Z / Z_{0}\right)$ with scale length $Z_{0}=0.04$ in our units, respectively. The cut off radius for mass density of the halo component is 1.2 in our units. Velocity dispersion of the halo component at a given point is set to be isotropic and given according to the virial theorem. 
In addition to the rotational velocity made by the gravitational field of disk and halo component, the initial radial and azimuthal velocity dispersion are given to disk component according to the epicyclic theory with Toomre's parameter (Binney \& Tremaine 1987) $Q=$ 1.0. This adopted value for $Q$ parameter is appreciably smaller compared with the value required for stabilizing the initial disk against the non-axisymmetric dynamical instability (e.g. bar instability). The reason for this adoption is that the initial disk is assumed to be composed mostly of interstellar gas and thus random kinetic energy in the disk is considered to be rather smaller because of gaseous dissipation in the disk. The vertical velocity dispersion at given radius is set to be 0.5 times as large as the radial velocity dispersion at that point, as is consistent with the observed trend of the Milky Way (e.g., Wielen 1977).

\subsubsection{Gas dynamics}

The collisional and dissipative nature of the interstellar medium (ISM) is modeled by the sticky particle method (Schwarz 1981). It should be emphasized here that this discrete cloud model can at best represent the real interstellar medium of galaxies in a schematic way. Actually, considerably complicated nature of interstellar medium, which can be modeled as three phase structure ('hot', 'warm', and 'cool' gas) by McKee \& Ostriker (1977), would not be so simply modeled by the 'sticky particle' method in which gaseous dissipation is modeled by ad hoc cloud-cloud collision: Any existing numerical method could not model the real interstellar medium in an admittedly proper way. More elaborated numerical modeling for real interstellar medium would be necessary for our further understanding of dynamical evolution in dissipative galaxy mergers. To mimic the galaxy mergers which are occurred at high redshift and thus very dissipative because of a considerably larger amount of interstellar gas in the progenitor disks, we assume that the fraction of gas mass in a disk is set to be 1.0 initially. The size of the clouds is set to be 5.0 
$\times 10^{-3}$ in our units unless specified. The radial and tangential restitution coefficient for cloud-cloud collisions are set to be 0.5 and 0.0 , respectively.

\subsubsection{Orbital configurations of galaxy mergers}

In all of the merger simulations, the orbit of the two disks is set to be initially in the $x y$ plane. The initial distance between the center of mass of the two disks, the pericenter distance, and the eccentricity of the merger orbit are 4.0, 1.0, and 1.0, respectively, for all models. The spin of each galaxy in a merger is specified by two angle $\theta_{i}$ and $\phi_{i}$, where suffix $i$ is used to identify each galaxy. $\theta_{i}$ is the angle between the $z$ axis and the vector of the angular momentum of a disk. $\phi_{i}$ is the azimuthal angle measured from $x$ axis to the projection of the angular momentum vector of a disk on to $x y$ plane. $\theta_{1}, \theta_{2}, \phi_{1}$, and $\phi_{2}$ are set to be 30.0, 120.0,90.0, and 0.0, respectively. The time when the progenitor disks merge

completely and reach the dynamical equilibrium is less than 15.0 in our units for most of models and does not depend so strongly on the history of star formation in the present calculations. In the present study, we do not describe the results of models other than those given above, principally because diversity in initial orbital conditions of galaxy merging only introduce a scatter in the present numerical results and does not drastically change the essence of the derived results, as has been already pointed out by Paper I. Furthermore we do not here intend to investigate the importance of multiple galaxy merging in the chemodynamical evolution of elliptical galaxies, which has been already investigated in Paper I. 


\subsubsection{Comparison with previous merger models}

Initial conditions of galaxy mergers adopted in the present study differ from those adopted in previous studies (e.g., Mihos \& Hernquist 1996) mainly in the following two points. Firstly, as is described in $§ 2.1 .1$, the present initial disk model does not include any remarkable bulge components, and accordingly corresponds to 'pure' late-type spiral. The reasons for this are firstly that by not including bulges, we can more clearly elucidate the dynamical effects of dissipative galaxy merging on chemical evolution of galaxies (that is, we can discriminate the effects of galaxy merging and those of galactic bulges), and secondly that extensive observational studies have not been yet accumulated which can give strong constraints on physical properties of galactic bulges in high redshift disks adopted in the present study. Although it is highly possible that galactic bulges greatly affect the chemical evolution of galaxy mergers, we investigate this issue in our future papers. Secondly, gas mass fraction adopted in the present study (1.0) is much larger that that of the observed typical values of the present disks ( $0.1 \sim 0.2$, adopted in previous studies). This is firstly because we now consider chemodynamical evolution of gas-rich mergers at high redshift, and secondly because we generally must begin with purely gaseous galactic disks for solving chemical evolution of galaxies. The main reason for our adoption of high redshift mergers is that the observed considerably tight color-magnitude relation of elliptical galaxies (Bower, Lucey, \& Ellis 1992; Ellis et al. 1997) and the relatively smaller redshift evolution of photometric properties of elliptical galaxies (Aragón-Salamanca et al. 1993; Franx \& van Dokkum 1996) imply relatively early formation of elliptical galaxies. Actually, the gas mass fraction in precursor disks of a merger is different between galaxy mergers and depends on the epoch of the merging. Although this difference could yield a great variety of chemical and dynamical structures in merger remnants, we do not intend to consider this important difference for simplicity in the present paper and will address this in our future papers. 
Furthermore we should emphasize here the following three less realistic assumptions or oversimplifications on initial conditions of galaxy mergers adopted in the present study. Firstly, in order to elucidate more clearly dynamical effects on chemophotometric evolution of galaxy mergers, we assume fully developed gas-rich exponential disks ('mature' disks) in investigating high redshift galaxy mergers. It is, however, not observationally clarified whether or not high redshift disk galaxies are so fully developed ('mature') as is assumed in the present study. Accordingly, the adopted assumption of 'mature' disks could correspond to oversimplification. Secondly, we neglect the importance of continuous gaseous infall onto galactic disks from external environments in the evolution of disk galaxies, which is expected from observational studies on the G-dwarf problem in the Galaxy (e.g., van den Bergh 1962) and star formation history of galaxies (e.g., Larson 1972; Larson, Tinsley, \& Caldwell 1980; Kennicutt 1983; Sandage 1986). Owing to this neglect of gaseous infall for merger progenitor disks, the present chemodynamical model suffers from overproduction of metal-poor stars in merger remnants, as is pointed out by other chemical evolution models (e.g., Tantalo et al. 1996). Thirdly, we assume that two disks merge with each other to form an elliptical when the disks still have a larger gas mass fraction $(\geq 0.2)$. Owing to this assumption, the dynamical effects of galaxy merging on ISM and thus on chemical evolution of ISM is more drastic in the present chemodynamical model. Actually, the epoch of major galaxy merging is probably widely spread, depending on orbit configurations of galaxy merging (impact parameters) and galaxy environments. Accordingly, it is equally possible that in gas-poor galaxy mergers (late mergers), galaxy merging does not induce the secondary starburst strongly enough to change substantially stellar populations of galaxy mergers. Because of these three apparently less realistic assumptions, we should interpret more carefully the present numerical results and consider the applicability of the results to real elliptical galaxies. These assumptions indeed may weakens the validity of the present chemodynamical model, however, we believe that even the present model enables 
us to grasp some essential ingredients of dynamical effects on chemophotometric evolution of galaxy mergers. Our future studies, which do not adopt the above three less realistic assumptions and instead include more realistic initial conditions of galaxy mergers within a plausible cosmological model, will help us to understand more deeply and thoroughly galactic chemophotometric evolution affected largely by dynamical evolution of galaxy mergers.

\subsection{Star formation model}

\subsubsection{Gas consumption rate}

Gas consumption by star formation in galaxy mergers is modeled by converting the collisional gas particles into collisionless new stellar ones according to the algorithm of star formation described below. We adopt the Schmidt law (Schmidt 1959) with exponent $\gamma$ $=2.0(1.0<\gamma<2.0$, Kennicutt 1989) as the controlling parameter of the rate of star formation. The amount of gas consumed by star formation for each gas particle in each time step, $\dot{M}_{\mathrm{g}}$, is given as:

$$
\dot{M}_{\mathrm{g}} \propto C_{\mathrm{SF}} \times\left(\rho_{\mathrm{g}} / \rho_{0}\right)^{\gamma-1.0}
$$

where $\rho_{\mathrm{g}}$ and $\rho_{0}$ are the gas density around each gas particle and the mean gas density at $0.48 R_{\mathrm{d}}$ of an initial disk, respectively. This star formation model is similar to that of Mihos, Richstone, \& Bothun (1992). In order to avoid a large number of new stellar particles with different mass, we convert one gas particle into one stellar one according to the following procedure. First we give each gas particle the probability, $P_{\text {sf }}$, that the gas

particle is converted into stellar one, by setting the $P_{\mathrm{sf}}$ to be proportional to the $\dot{M}_{\mathrm{g}}$ in equation (1) estimated for the gas particle. Then we draw the random number to determine whether or not the gas particle is totally converted into one new star. This method of star 
formation enables us to control the rapidity of star formation without increase of particle number in each simulation thus to maintain the numerical accuracy in each simulation. The $C_{\mathrm{SF}}$ is meant to be proportional to the ratio of dynamical time-scale of the system to the time-scale of gas consumption by star formation. We accordingly emphasize that even if the coefficient of the Schmidt law is constant for galaxies, values of $C_{\mathrm{SF}}$ can be different between galaxies owing to the difference in dynamical time-scale between galaxies. The $C_{\mathrm{SF}}$ is considered to depend exclusively on galactic disk mass $\left(M_{\mathrm{d}}\right)$, as is described later. The equation (1) furthermore states that a larger number of stellar particles are created at the regions where the local gas density becomes larger owing to the onset of local Jeans instability. The positions and velocity of the new stellar particles are set to be the same as those of original gas particles.

\subsubsection{Supernovae feedback effects}

Thermal and dynamical (kinematic) heating of ISM driven by supernovae are found to affect greatly the formation and evolution of galaxies (e.g., Katz 1992; Navarro \& White 1993). We here consider the dynamical feedback effects of Type Ia (SNIa) and Type II supernovae (SNII) and neglect the feedback effects of thermal heating on galaxy evolution. The reason for this neglect is firstly that our gaseous model does not solve thermal evolution of ISM, and secondly that such thermal effects is found to be less important than dynamical feedback effects owing to efficient cooling of ISM (e.g., Katz 1992; Navarro \& White 1993). Accordingly some fraction of total energy ejected from SNIa and SNII is assumed to give velocity perturbation of ISM in the present study. The details of the supernovae feedback

effects are given as follows. Total energy produced by SNIa and SNII at the time $t$ for each $i$ th newly born stellar particle $\left(\Delta E_{\mathrm{sn}, i}(t)\right)$ is given as,

$$
\Delta E_{\mathrm{sn}, i}(t)=m_{\mathrm{s}, i} S_{i}\left(t-t_{i}\right)
$$


where $m_{\mathrm{s}, i}$ is the mass of the $i$ th stellar particle, $S_{i}\left(t-t_{i}\right)$ is the total energy of SNI and SNIIa per unit mass at the time $t$, and $t_{i}$ represents the time when the $i$ th stellar particle is born from a gas particle. The above formalism is more elaborated than previous studies in the following two points. Firstly, we consider time-delay between the epoch of star formation and the epoch of supernovae explosions for stars with variously different masses. In general, life-time of stars depends on stellar mass in such a way that more massive stars die earlier than less massive ones. Consequently, the time-delay is different between stars with different masses and total number of SNIa and SNII is also time-dependent. Since each $i$ th stellar particle $\left(\sim 10^{6} M_{\odot}\right.$ in the present study) is assumed to consist of stars with different masses, the $S_{i}\left(t-t_{i}\right)$ is time-dependent. Secondly, we consider that both the energy ejected from SNIa and that from SNII can be returned to ISM of galaxy mergers but neglect thermal energy ejected from long-lived evolved stars. The total energy per a supernovae is assumed to be $10^{51} \mathrm{erg}$ both for SNIa and SNII. The typical epoch of supernovae explosions are different between these two types of supernovae $\left(\sim 10^{9}\right.$ yr for $\mathrm{SNIa}$ and $\sim 10^{7} \mathrm{yr}$ for SNII), and this difference is reflected on the time-dependence of the $S_{i}\left(t-t_{i}\right)$.

Some fraction of the $\Delta E_{\mathrm{sn}, i}(t)$ are assumed to be returned to gas particles (ISM) and then to change kinetic energy of gas particles surrounding $i$ th stellar particle. Each $j$ th gas particle around $i$ th stellar particle can receive a velocity perturbation $\left(\Delta v_{\mathrm{sn}, j}\right)$ directed radially away from the $i$ th stellar particle. The $\Delta v_{\mathrm{sn}, j}$ at the time $t$ satisfies the following relation:

$$
f_{\mathrm{sn}} \Delta E_{\mathrm{sn}, i}(t)=1 / 2 \sum_{j}^{N_{\mathrm{nei}, i}} m_{\mathrm{g}, j}\left(\Delta v_{\mathrm{sn}, j}\right)^{2},
$$

where $f_{\mathrm{sn}}$ represents the fraction of supernovae energy returned to ISM, $N_{\text {nei, } i}$ is total number of gas particles that are located within 0.05 (in our units) around $i$ th stellar particle, and $m_{\mathrm{g}, j}$ is a mass of each $j$ th gas particle. This formulation is similar to that adopted in Navarro \& White (1993). It depends largely on the parameter $f_{\text {sn }}$ how greatly 
SNIa and SNII can provide dynamical (kinetic) feedback effects on ISM of galaxy mergers. It is, however, not observationally clarified which values of $f_{\mathrm{sn}}$ is the most plausible and realistic for the evolution of ISM affected by SNIa and II feedback. Thus we investigate the importance of the parameter $f_{\text {sn }}$ by varying values of $f_{\text {sn }}\left(0.0 \leq f_{\text {sn }} \leq 0.5\right.$ in the present study).

All the calculations related to the above dynamical evolution including the dissipative dynamics, star formation, SNIa and SNII feedback effects, and gravitational interaction between collisionless and collisional component have been carried out on the GRAPE board (Sugimoto et al. 1990) at Astronomical Institute of Tohoku University. The number of particles for a galaxy merger model is 10000 for the halo component and 20000 for the gaseous component. The parameter of gravitational softening is set to be fixed at 0.04 in all the simulations. The time integration of the equation of motion is performed by using 2-order leap-flog method. Energy and angular momentum are conserved within 1 percent accuracy in a test collisionless merger simulation. Most of the calculations are set to be stopped at $\mathrm{T}=20.0$ in our units unless specified.

\subsection{Chemophotometric model}

Photometric and spectroscopic properties such as global colors and metallicity gradient in elliptical galaxies depend critically on how the metallicity and age of stellar components are distributed in galaxies. Accordingly, we must analyze the distribution of stellar age and that of stellar metallicity in merger remnants for each model in order to grasp some fundamental characteristics of chemical, photometric, and spectroscopic properties in merger remnants. We calculate age and metallicity distribution, based on the age $\left(t_{i}\right)$ and

metallicity $\left(Z_{i}\right)$ assigned to each $i$ th stellar particle, as described in detail later. The outline for this calculation is as follows. First we derive the distribution of stellar age and that 
of stellar metallicity by assigning age and metallicity to stellar particles according as the law of chemical enrichment applied to this study. Next, by using the stellar population synthesis method, we calculate the photometric and spectroscopic properties based on the derived distribution of age and metallicity in merger remnants.

\subsubsection{Chemical enrichment}

Chemical enrichment through star formation during galaxy merging is considered to proceed locally and inhomogeneously. In the present study, we investigate time-evolution of five species of chemical components, $\mathrm{H}, \mathrm{He}, \mathrm{O}, \mathrm{Mg}$, and $\mathrm{Fe}$ as well as the conventionally used mean metallicity $Z$. The mean metallicity of $Z$ for each $i$ th stellar particle is represented by $Z_{i}$. Total mass of each $j$ th $(j=1,2,3,4$,and 5$)$ chemical component $(\mathrm{H}, \mathrm{He}, \mathrm{O}, \mathrm{Mg}$, and $\mathrm{Fe}$ ) ejected from each $i$ th stellar particle at the time $t$ is given as,

$$
\Delta z_{i, j}(t)=m_{\mathrm{s}, i} Y_{i, j}\left(t-t_{i}\right)
$$

where $m_{\mathrm{s}, i}$ is the mass of the $i$ th stellar particle, $Y_{i, j}\left(t-t_{i}\right)$ is the mass of each $j$ th chemical component ejected from stars per unit mass at the time $t$, and $t_{i}$ represents the time when the $i$ th stellar particle is born from a gas particle. The $\Delta z_{i, j}(t)$ is given to neighbor gas particles locating within $R_{\text {chem }}$ from the position of the $i$ th stellar particle. This $R_{\text {chem }}$ is referred to as chemical mixing length in the present paper, and represents the region within which the neighbor gas particles are polluted by metals ejected from stellar particles. The value of $R_{\text {chem }}$ relative to the typical size of a galaxy could be different between galaxies, accordingly the value of $R_{\text {chem }}$ is considered to be a free parameter in the present study. The value of $R_{\text {chem }}$ examined the most extensively in the present study is 0.1 , which corresponds to the half of the scale-length of initial disks. Initial gaseous metallicity $(Z)$ is set to be 0.1 solar, which is exactly the same as that of infall gas adopted in the chemical evolution of disk galaxies with gaseous infall (e.g., Matteucci \& Fran@is 1989). We adopt this initial 
enrichment of ISM in disks in order to avoid overproduction of less metal-enriched stellar components that are not allowed for plausible chemical evolution of disk galaxies (e.g., van den Bergh 1962). Initial value of the abundance ratio $[\mathrm{Mg} / \mathrm{Fe}]$ for ISM in disks is set to be 0.8, which is similar to a typical value of metal-poor halo ISM that is metal-enriched mostly by SNII and a candidate for infall gas of disk galaxies (e.g., Matteucci \& Franoiis 1989).

Since we now consider the time-delay between the epoch of star formation and that of supernovae explosions, the mass of each $j$ th chemical component ejected from each $i$ th stellar particle, which are basically determined by $Y_{i, j}\left(t-t_{i}\right)$, is also strongly time-dependent. We estimate the mass-dependent life-time of stars that becomes SNIa or SNII by using mass-age relation given by Bressan et al. (1993). The fraction of close binary stars of SNIa relative to SNII (represented by A parameter in Matteucci \& Tornambè 1987) is assumed to be 0.1 . The $Y_{i, j}\left(t-t_{i}\right)$ furthermore depends on stellar yields, IMF profiles, upper cutoff mass $M_{\text {up }}$, and lower one $M_{\text {low }}$. In the present study, we adopt the Salpeter $\mathrm{IMF}, \phi(m) \propto m^{-1.35}$, with $M_{\mathrm{up}}=120 M_{\odot}$ and $M_{\mathrm{low}}=0.6 M_{\odot}$. The reason for this larger $M_{\text {low }}\left(=0.6 M_{\odot}\right)$ is essentially because we do not have stellar yield tables for stars with masses less than 0.6. To calculate the ejected mass of gas and metals in $Y_{i, j}\left(t-t_{i}\right)$, we use stellar yields derived by Woosley \& Weaver (1995) for SNII, Nomoto, Thielemann, \& Yokoi (1984) for SNIa, and Bressan et al. (1993) and Magris \& Bruzual (1993) for low and intermediate mass stars. More details of the time-dependence of $Y_{i, j}\left(t-t_{i}\right)$ for a given IMF, $M_{\text {up }}$, and $M_{\text {low }}$ are given in Appendix A.

\subsubsection{Population synthesis}

It is assumed in the present study that the spectral energy distribution (SED) of a model galaxy is a sum of the SED of stellar particles. The SED of each ith stellar particle is assumed to be a simple stellar population (SSP) that is a coeval and chemically 
homogeneous assembly of stars. Thus the monochromatic flux of a galaxy with age $t, F_{\lambda}(t)$, is described as;

$$
F_{\lambda}(t)=\sum_{\mathrm{star}} F_{\mathrm{SSP}, \lambda}\left(Z_{i}, \tau_{i}\right) \times m_{\mathrm{s}, i}
$$

where $F_{\mathrm{SSP}, \lambda}\left(Z_{i}, \tau_{i}\right)$ and $m_{\mathrm{s}, i}$ are a monochromatic flux of SSP of age $\tau_{i}$ and metallicity $Z_{i}$, where suffix $i$ identifies each $i$ th stellar particle, and mass of each stellar particle, respectively. The age of SSP, $\tau_{i}$, is defined as $\tau_{i}=t-t_{i}$, where $t_{i}$ is the time when a gas particle is converted into a stellar one. The metallicity of SSP is exactly the same as that of the stellar particle, $Z_{i}$, and the summation $\left(\sum\right)$ in equation $(5)$ is done for all stellar particles in a model galaxy. A stellar particle is assumed to be composed of stars whose age and metallicity are exactly the same as those of the stellar particle and the total mass of the stars is set to be the same as that of the stellar particle. Thus the monochromatic flux of SSP at a given wavelength is defined as

$$
F_{\mathrm{SSP}, \lambda}\left(Z_{i}, \tau_{i}\right)=\int_{M_{\mathrm{low}}}^{M_{\mathrm{up}}} \phi(M) f_{\lambda}\left(M, \tau_{i}, Z_{i}\right) d M,
$$

where $M$ is mass of a star, $f_{\lambda}\left(M, \tau_{i}, Z_{i}\right)$ is a monochromatic flux of a star with mass $M$, metallicity $Z_{i}$ and age $\tau_{i}$, and $\phi(M)$ is a initial mass function (IMF) of stars. In this paper, we use the $F_{\mathrm{SSP}, \lambda}\left(Z_{i}, \tau_{i}\right)$ of GISSEL96 which is a latest version of Bruzual \& Charlot (1993).

\section{EDITOR: PLACE TABLE ?? HERE.}

\subsection{Main points of analysis}

We mainly investigate how initial galactic disk mass $\left(M_{\mathrm{d}}\right)$ determines chemical, photometric, and spectroscopic evolution of galaxy mergers by varying values of the parameter $M_{\mathrm{d}}$. In the present study, we assume Freeman's law (Freeman 1970), $M_{\mathrm{d}} \propto R_{\mathrm{d}}{ }^{2}$, and constant initial ratio of dark matter to disk mass $\left(M_{\mathrm{d}} / M_{\mathrm{t}} \sim\right.$ constant $)$. Hence if 
we give $M_{\mathrm{d}}$, then $R_{\mathrm{d}}, v$, and $t_{\mathrm{dyn}}$ are automatically determined $\left(R_{\mathrm{d}}, v\right.$, and $t_{\mathrm{dyn}}$ are described as a function of $M_{\mathrm{d}}$.). We here adopt $M_{\mathrm{d}}=6.0 \times 10^{10} M_{\odot}, R_{\mathrm{d}}=17.5 \mathrm{kpc}$, $v=1.21 \times 10^{2} \mathrm{~km} / \mathrm{s}$, and $t_{\mathrm{dyn}}=1.41 \times 10^{8} \mathrm{yr}$ as reference values, which are exactly the same as those in Paper I. The $M_{\mathrm{d}}$ dependence of $R_{\mathrm{d}}, v$, and $t_{\mathrm{dyn}}$ are accordingly described as: $R_{\mathrm{d}}=17.5\left(M_{\mathrm{d}} / 6.0 \times 10^{10} M_{\odot}\right)^{1 / 2} \mathrm{kpc}, v=1.21 \times 10^{2}\left(M_{\mathrm{d}} / 6.0 \times 10^{10}\right)^{1 / 4} \mathrm{~km} / \mathrm{s}$, $t_{\mathrm{dyn}}=1.41 \times 10^{8}\left(M_{\mathrm{d}} / 6.0 \times 10^{10}\right)^{1 / 4} \mathrm{yr}$. We here emphasize that the adopted scaling is only fiducial, thus absolute magnitude of the present results based on this scaling can be changed if other types of galactic scaling are adopted. $M_{\mathrm{d}}$ is considered to be the most important parameter in the present study, principally because $M_{\mathrm{d}}$ can control the following two physical quantities that greatly affect galactic evolution. The first is the rapidity of star formation, which means the ratio of dynamical time-scale to star formation one represented by $C_{\mathrm{SF}}$ in $\S 2.2 .1$. The second is that the effectiveness of supernovae feedback, which means the ratio of total energy expected to be ejected from supernovae (in the course of galaxy evolution) to total potential energy in a galaxy (hereafter represented by $f_{\text {eff }}$ ). The mass-dependent properties of galaxy mergers, which are described later (§3), are due essentially to mass-dependence of these two quantities $\left(C_{\mathrm{SF}}\right.$ and $\left.f_{\text {eff }}\right)$ in the present study. The expected $M_{\mathrm{d}}$ dependence of $C_{\mathrm{SF}}$ and $f_{\text {eff }}$ are $C_{\mathrm{SF}} \propto\left(M_{\mathrm{d}}\right)^{0.25}$ and $f_{\text {eff }} \propto\left(M_{\mathrm{d}}\right)^{-0.5}$. Accordingly, if we set both the value of $C_{\mathrm{SF}}$ and that of $f_{\text {eff }}$ in the model with $M_{\mathrm{d}}=10^{10} M_{\odot}$ to be 1.0, the ranges of the two quantities are $1.0 \leq C_{\mathrm{SF}} \leq 3.16$ and $1.0 \leq f_{\mathrm{eff}}{ }^{-1} \leq 4.0$ for galaxy mergers with $10^{10} M_{\odot} \leq M_{\mathrm{d}} \leq 10^{12} M_{\odot}$. More details of these dependence are given in Appendix B.

We particularly investigate $M_{\mathrm{d}}$ dependence of mean and radail properties of stellar metallicity (Fe, $\mathrm{Mg}$, and $\mathrm{Z})$, the abundance ratio of $[\mathrm{Mg} / \mathrm{Fe}]$, global colors $(U-R, B-R$, and $V-K)$, and line indices $\left(\mathrm{H}_{\beta}, \mathrm{Mg}_{2}\right.$, and $\left.\langle F e\rangle\right)$, mass-to-light-ratio $\left(M / L_{B}\right.$ and $\left.M / L_{K}\right)$. Here $\langle F e\rangle$ represents the mean value of Fe5270 and Fe5335 (Faber et al. 1995). The values of $M_{\mathrm{d}}$ range from $10^{10} M_{\odot}$ to $10^{12} M_{\odot}$ in the present merger models. We furthermore 
investigate fundamental roles of chemical mixing length $\left(0.1 \leq R_{\text {chem }} \leq 0.4\right)$ and fraction of supernovae energy that is returned to ISM $\left(0.0 \leq f_{\mathrm{sn}} \leq 0.5\right)$. In total, 13 model are investigated, including models with different masses (Model $1 \sim 6$ ), those with no supernova feedback (Model 7, 8, and 9), those with larger chemical mixing length (Model 10 and 11), and those with no gaseous dissipation (Model 12 and 13). The values of parameters and brief summary of the results are given in Table 1. First, second, third, and fourth columns in the Table 1 denote the model number, the value of $M_{\mathrm{d}}$, that of $R_{\text {chem }}$, and that of $f_{\text {sn }}$ respectively, for each model. Fifth, sixth, seventh, eighth, ninth, and tenth columns give mean stellar metallicity $\left(\left\langle Z_{*}\right\rangle\right)$, the abundance ratio of $[\mathrm{Mg} / \mathrm{Fe}]$, global $V-K$ color (in units of mag), $\mathrm{Mg}_{2}(\mathrm{mag}), \mathrm{H}_{\beta}(\AA)$, and $\langle\mathrm{Fe}\rangle$ line indices $(\AA)$ in the central region of merger remnants (within $0.1 R_{\mathrm{e}}$ ). Mass-to-light ratio, which means the ratio of total stellar mass to stellar luminosity, is given in eleventh column for $B$-band $\left(M / L_{\mathrm{B}}\right)$ and in twelfth one for $K$-band $\left(M / L_{\mathrm{K}}\right)$. Thirteen column denotes the value of metallicity gradient $\left(\Delta \log \left\langle Z_{*}\right\rangle / \Delta \log R\right)$. Comments for each model are given in the fourteenth column. As is described above, our main points of the present study are not the origins of structural and kinematical properties characteristics of ellipticals. Therefore we do not here intend to discuss these properties. More details on fundamental structural and kinematical properties of merger remnants are given in Bekki \& Shioya (1997) and our Paper I. Lastly, it should be stressed that although the relative importance of each parameter in determining galactic properties derived in the present study (e.g., $M_{\mathrm{d}}$ dependence of chemophotometric properties of merger remnants) is considerably meaningful, the absolute magnitude of the derived galactic properties should be more carefully interpreted: This is primarily because the present chemodynamical model is still rather idealized in terms of dissipative dynamics, star formation model, time-independent IMF, chemical enrichment processes of galaxy mergers, and galaxy scaling relations. 
EDITOR: PLACE FIGURE 1 HERE.

EDITOR: PLACE FIGURE 2 HERE.

EDITOR: PLACE FIGURE 3 HERE.

\section{Results}

Firstly, we describe how initial disk mass of galaxy mergers determines fundamental chemical, photometric, and spectroscopic properties of merger remnants in $§ 3.1$. Secondly, we emphasize the importance of supernova feedback effects in the formation of spectrophotometric relationships observed in elliptical galaxies in $\S 3.2$. Thirdly, we provide fundamental roles of parameters other than galactic mass in determining spectrophotometric properties of merger remnants in $\S 3.3$.

\subsection{Dependence on initial disk mass of galaxy mergers}

\subsubsection{Metallicity distribution}

Figure 1 shows time-evolution of gas mass fraction $\left(f_{\mathrm{g}}\right)$ and that of mean stellar metallicity $\left(\left\langle Z_{*}\right\rangle\right)$ in galaxy mergers for Model 1 with $M_{\mathrm{d}}=10^{10} M_{\odot}$ and Model 2 with $M_{\mathrm{d}}=10^{12} M_{\odot}$. A larger amount of ISM is finally converted into stellar components in a more massive galaxy merger, firstly because a smaller amount of ISM is tidally stripped away from the merger owing to more rapid star formation, and secondly because the supernovae feedback does not so strongly suppress the formation of higher gaseous density 
region that is a potential site of efficient star formation in the merger. Consequently, a more massive galaxy merger has larger mean stellar metallicity, as is shown in Figure 1. This dependence of mean stellar metallicity on galactic mass is consistent with that derived in our Paper I. Figure 2 describes final metallicity distributions of stellar components $\left(Z_{*}\right)$ in merger remnants for Model 1 with $M_{\mathrm{d}}=10^{10} M_{\odot}$ and Model 2 with $M_{\mathrm{d}}=10^{12} M_{\odot}$. The peak of the metallicity distribution is located in the righter side for a more massive merger remnant, which means a larger amount of stellar components is substantially metal-enriched in the remnant. Figure 3 gives time evolution of stellar metallicity, $\left\langle Z_{*, \mathrm{Fe}}\right\rangle,\left\langle Z_{*, \mathrm{Mg}}\right\rangle$ and that of stellar abundance ratio of $[\mathrm{Mg} / \mathrm{Fe}]$. As is shown in Figure 3, both $\left\langle Z_{*, \mathrm{Fe}}\right\rangle$ and $\left\langle Z_{*, \mathrm{Mg}}\right\rangle$ are finally discernibly larger for a more massive merger. This is essentially because for the massive galaxy merger, owing to less effective supernova feedback, a larger amount of ISM can be converted into stellar components during dissipative galaxy merging and thus more metal-enriched with $\mathrm{Mg}$ and Fe. Furthermore the stellar abundance ratio of $[\mathrm{Mg} / \mathrm{Fe}]$ is slightly smaller for the more massive merger remnant. It should be emphasized here that $[\mathrm{Mg} / \mathrm{Fe}]$ is larger than the solar ratio $([\mathrm{Mg} / \mathrm{Fe}]=0.0)$ for the two merger models. This result reflects the fact that in the present merger model, two gas-rich disks are assumed to merge with each other at high redshift universe, where merger progenitor disks have a larger amount of less-metal enriched ISM.

EDITOR: PLACE FIGURE 1 HERE.

EDITOR: PLACE FIGURE 5 HERE.

EDITOR: PLACE FIGURE 6 HERE.

EDITOR: PLACE FIGURE 7 HERE. 


\subsubsection{Radial gradients}

The present merger model demonstrates that chemical evolution of ISM in galaxy merges proceeds in an very inhomogeneous way: In the central part of mergers, metal-enrichment is more efficient owing to radial inflow of metal-enriched ISM and the subsequent star formation triggered by dissipative galaxy merging, whereas in the outer part, metal-enrichment is less efficient owing to a larger amount of metal-enriched ISM tidally stripped away from mergers. As a natural result of this inhomogeneous chemical mixing in dissipative galaxy merging, both stellar and gaseous components have a number of interesting chemical (and spectrophotometric) properties in merger remnants. Among these, we here restrict ourselves to radial properties of stellar populations, and accordingly other important roles of inhomogeneous chemical mixing in determining fundamental properties of elliptical galaxies are described in $§ 4.2$. Figure 4 describes radial distribution of stellar metallicity $\left(\left\langle Z_{*}\right\rangle,\left\langle Z_{*, \mathrm{Fe}}\right\rangle\right.$, and $\left.\left\langle Z_{*, \mathrm{Mg}}\right\rangle\right)$ and that of the abundance ratio of $[\mathrm{Mg} / \mathrm{Fe}]$ in merger remnants for Model 1 with $M_{\mathrm{d}}=10^{10} M_{\odot}$ and Model 2 with $M_{\mathrm{d}}=10^{12} M_{\odot}$. Irrespectively of galactic mass, merger remnants show negative metallicity gradients fitted well by power-low profile (especially for the region $0.5 \leq R / R_{\mathrm{e}} \leq 5.0$ ). The metallicity gradients $\left(\Delta \log \left\langle Z_{*}\right\rangle / \Delta \log R\right)$ are steeper in the more massive merger remnant for the region $0.1 \leq R / R_{\mathrm{e}} \leq 1.0: \Delta \log \left\langle Z_{*}\right\rangle / \Delta \log R$ is -0.03 for Model 1 and -0.13 for Model 2 . This dependence is true for radial gradients of $\left\langle Z_{*, \mathrm{Fe}}\right\rangle$ and $\left\langle Z_{*, \mathrm{Mg}}\right\rangle$. The physical reason for this mass-dependence of metallicity gradients is principally that for a more massive galaxy merger, supernova feedback does not so strongly suppress the inward transfer of more metal-enriched ISM during dissipative galaxy merging and accordingly more metal-enriched ISM can be transferred to the central region of the merger and converted into new stellar components there. The derived dependence of stellar metallicity gradients on galactic mass is considerably different from that described in our Paper I, in which supernova feedback effects are not included at all. This difference between the present study and our Paper 
I strongly suggests that supernova feedback effects are critically important for the origin of stellar metallicity gradients of elliptical galaxies. The radial gradient of $[\mathrm{Mg} / \mathrm{Fe}]$, on the other hand, shows flat profile in the inner regions of merger remnants for the two models. Figure 5 shows the radial gradient of mean epoch of star formation $\left(\left\langle T_{\mathrm{SF}}\right\rangle\right)$ in merger remnants for Model 1 with $M_{\mathrm{d}}=10^{10} M_{\odot}$ and Model 2 with $M_{\mathrm{d}}=10^{12} M_{\odot}$. Here, a larger value of $\left\langle T_{\mathrm{SF}}\right\rangle$ at a given point means that stellar populations at the point are younger in average. Considering the results in Figure 4, Figure 5 clearly demonstrates that irrespectively of galactic mass, stellar populations of merger remnants are both younger and metal-enriched in the central part of merger remnants. This result reflects the fact that in the central part of galaxy mergers, more metal-enriched ISM that is transferred to galactic nuclei owing to efficient gas fueling in the later stage of galaxy merging can be preferentially and efficiently converted into stellar components within the deeper potential well. These younger and more metal-enriched stellar populations in the central part of merger remnants are consistent reasonably well with recent observational results of elliptical galaxies by Faber et al (1995).

Figure 6 describes radial gradients of integrated colors $(U-R, B-R$, and $V-K)$ in merger remnants for Model 1 and 2. The color gradients $(\Delta U-R / \Delta \log R, \Delta B-R / \Delta \log R$, $\Delta V-K / \Delta \log R)$ are more likely to be steeper in the more massive merger remnant for the region $0.1 \leq R / R_{\mathrm{e}} \leq 1.0$ : For example, $\Delta U-R / \Delta \log R$ is -0.07 for Model 1 and -0.13 for Model 2. This mass-dependence results from the already derived dependence that more massive merger remnants have larger metallicity gradients. The derived color gradients are appreciably shallower than mean values of those observed by Peletier, Valentijn, \& Jameson (1990) and Peletier et al. (1990). We here emphasize that if we measure the color gradients for the region $1.0 \leq R / R_{\mathrm{e}} \leq 10.0$, even color gradients of less massive merger remnants can become larger owing to considerably less metal-enriched stellar populations in the outer part of the merger remnants. Figure 7 gives radial gradients of three line indices $\left(\mathrm{H}_{\beta}, \mathrm{Mg}_{2}\right.$, 
and $\langle\mathrm{Fe}\rangle)$ in merger remnants for Model 1 and 2. The radial gradients of $\Delta \mathrm{H}_{\beta} / \Delta \log R$, $\Delta \mathrm{Mg}_{2} / \Delta \log R$, and $\Delta\langle\mathrm{Fe}\rangle / \Delta \log R$ for the region $0.1 \leq R / R_{\mathrm{e}} \leq 1.0$ are $0.036,-0.013$, and -0.121 , respectively, for Model 1 , and $0.051,-0.025$, and -0.250 , respectively, for Model

2. The physical reason for the positive gradient in $\mathrm{H}_{\beta}$ line index is that stellar populations in the outer part of merger remnants are considerably less metal-enriched. The derived gradients of line indices are appreciably shallower than mean values of those observed by Davies, Sadler, \& Peletier (1990). Moreover the radial gradients of $\mathrm{Mg}_{2}$ and $\langle\mathrm{Fe}\rangle$ are steeper for a more massive merger remnant, which means that a larger amount of metals ejected from SNIa and SNII are transferred to the central part of the remnant owing to more efficient fueling of metal-enriched ISM for the more massive galaxy merger.

\section{EDITOR: PLACE FIGURE 8 HERE.}

EDITOR: PLACE FIGURE 9 HERE.

\section{EDITOR: PLACE FIGURE 10 HERE.}

\subsection{Supernova feedback effects and spectrophotometric properties of merger remnants}

Here in $§ 3.2$, we present dependence of spectrophotometric properties of merger remnants on the parameter $f_{\mathrm{sn}}$. We compare the results of models with $f_{\mathrm{sn}}=0.0$ (no supernova feedback models, Model 7, 8, and 9) with those of models with $f_{\mathrm{sn}}=0.1$ and 0.5 (Model $1 \sim 6$ ) and thereby observe how the supernova feedback effects control fundamental characteristics of spectrophotometric properties in merger remnants. In particular, we 
describe the importance of the supernova feedback effects in the formation of the following three observational spectrophotometric relationships of elliptical galaxies: (1) The CM relation, (2) The $\mathrm{Mg}_{2}-\sigma$ relation, and (3) The luminosity-dependence of mass-to-light ratio. We here focus on rather qualitative behaviors of the derived results, and accordingly the qualitative comparison of the present results with observational ones is given in $§ 4.1$.

\subsubsection{The CM relation}

Figure 8 shows the dependence of $U-R, B-R$, and $V-K$ colors on $B$ band luminosity $\left(L_{\mathrm{B}}\right)$ in merger remnants for models with different initial disk mass $\left(M_{\mathrm{d}}=10^{10}, 10^{11}, 10^{12} M_{\odot}\right)$ and strength of supernovae feedback $\left(f_{\mathrm{sn}}=0.0,0.1,0.5\right)$. We can clearly observe the color-magnitude relation of merger remnants for each color $(U-R$, $B-R$, and $V-K)$ in Figure 8: More luminous merger remnants (ellipticals) have redder colors. The primary reason for the derived color-magnitude relation of merger remnants is that more massive (luminous) galaxy mergers can have larger mean stellar metallicity owing to more efficient star formation and chemical enrichment: The CM relation derived in the present study is due to mass-metallicity relation of merger remnants. This result implies that the existence of the observed CM relation is not necessarily inconsistent with the merger scenario of elliptical galaxy formation, furthermore that the origin of the observed CM relation can be closely associated with dissipative galaxy merging. As is observed in Figure 8, the difference of colors between merger remnants with different luminosity can be more discernable in the models with $f_{\mathrm{sn}}=0.5$ than in the models with $f_{\mathrm{sn}}=0.0$ (no feedback). This result suggests that in addition to the rapidity of star formation (the

importance of which is described in the Paper I), supernova feedback effects play a vital role in reproducing the CM relation of elliptical galaxies. Thus more luminous ellipticals formed by galaxy mergers between more massive spirals show redder colors, principally because 
more massive mergers suffer less severely from supernovae feedback effects thus have more metal-enriched stellar populations.

\subsubsection{The $\mathrm{Mg}_{2}-\sigma$ relation}

Figure 9 gives the dependence of central $\mathrm{Mg}_{2}$ index (within $0.1 R_{\mathrm{e}}$ ) on the central velocity dispersion in merger remnants for models with different initial disk mass $\left(M_{\mathrm{d}}=10^{10}, 10^{11}, 10^{12} M_{\odot}\right)$ and strength of supernovae feedback $\left(f_{\mathrm{sn}}=0.0,0.1,0.5\right)$. As is observed in Figure 9, although there is no clear trend in $\mathrm{Mg}_{2}-\sigma$ relation for models with $f_{\mathrm{sn}}=0.0$ (no supernova feedback), $\mathrm{Mg}_{2}-\sigma$ relation which is qualitatively consistent with that observed in elliptical galaxies is more discernable for models with $f_{\text {sn }}=0.1$ and 0.5. This result clearly demonstrates that the origin of the observed $\mathrm{Mg}_{2}-\sigma$ relation of elliptical galaxies can be due partly to physical processes associated with SNIa and SN II events. The reason for the clearer $\mathrm{Mg}_{2}-\sigma$ relation in models with $f_{\mathrm{sn}}=0.1$ and 0.5 is essentially that for more massive galaxy mergers, a larger amount of more metal-enriched ISM can be transferred to the central part of the mergers with deeper potential wells. Moreover the $\mathrm{Mg}_{2}-\sigma$ relation derived in models with $f_{\mathrm{sn}}=0.5$ is more stepper and thus similar to the observational one than that derived in models with $f_{\mathrm{sn}}=0.1$. This result implies that stronger supernovae feedback effects can be required for more successful reproduction of the observed $\mathrm{Mg}_{2}-\sigma$ relation. Thus, the present chemodynamical model suggests that more

massive ellipticals with larger central velocity dispersion formed by galaxy mergers between more massive gas-rich spirals are more likely to show larger central $\mathrm{Mg}_{2}$ index. 


\subsubsection{The mass-to-light-ratio}

Figure 10 shows dependence of 'mass-to-light-ratio' (in $B$ and $K$ bands) on luminosity in merger remnants for models with different initial disk mass $\left(M_{\mathrm{d}}=10^{10}, 10^{11}, 10^{12} M_{\odot}\right)$ and strength of supernovae feedback $\left(f_{\mathrm{sn}}=0.0,0.1,0.5\right)$. Here the 'mass-to-light-ratio' means the ratio of total stellar mass to total stellar luminosity, thus does not mean the ratio of total mass (stellar mass and dark matter mass) to total stellar luminosity. As is shown in Figure 10, mass-to-light ratio in $B$ band $\left(M / L_{B}\right)$ is larger for more luminous merger remnants whereas that in $K$ band $\left(M / L_{K}\right)$ is smaller for more luminous merger remnants. The essential reason for these dependences is that the more luminous merger remnants have larger mean stellar metallicity owing to more efficient chemical evolution of galaxy mergers. Furthermore, the luminosity dependence of mass-to-light-ratio can be more discernibly observed in the models with $f_{\mathrm{sn}}=0.1$ and 0.5 than in those with $f_{\mathrm{sn}}=0.0$ (no feedback effects). This is primarily because the difference in mean stellar metallicity between galaxy mergers with different masses is larger in models with $f_{\mathrm{sn}}=0.1$ and 0.5 than in those with $f_{\mathrm{sn}}=0.0$. Assuming that the observed mass-to-light ratio inferred from the FP is the ratio of stellar mass to stellar luminosity (not the ratio of total mass to stellar luminosity), the derived dependence of $M / L_{B}$ on $L_{B}$ is only qualitatively consistent with that inferred from the FP of elliptical galaxies (e.g., Djorgovski \& Davis 1987; Dressler et al. 1987; Bender et al. 1992; Pahre, de Carvalho, \& Djorgovski 1998). This result implies that the effect of stellar population alone (that is, the mass-metallicity relation) can not explain the origin of the $B$ band FP fully. Furthermore the derived dependence of $M / L_{K}$ on $L_{K}$ is obviously inconsistent with that inferred from the $K$ band FP (e.g., Djorgovski, Pahre, \& de Carvalho 1996). This result suggests that the origin of the observed dependence of $M / L_{K}$ on $L_{K}$ cannot be explained at all by the differences in stellar populations between elliptical galaxies with different masses. 
The origin of the $K$ band FP has been already demonstrated not to be explained so simply by some effects of stellar population (Pahre \& Djorgovski 1997). The dependence of the mass-to-light ratio on mass inferred from the $K$ band FP is furthermore suggested to be explained by structural and kinematical nonhomology in elliptical galaxies (Djorgovski et al. 1996). Actually, numerical simulations on dissipationless galaxy mergers (Capelato, de Carvalho \& Carlberg 1995) and dissipative ones (Bekki 1998) demonstrated that structural and kinematical nonhomology is a natural result of violent relation combined with gaseous dissipation in galaxy merging. These observational and theoretical studies imply that the above failure in the successful reproduction of the $K$ band FP does not point out one of serious disadvantages of the present merger model but is on line with the early suggestion that the $K$ band FP should be explained by the supposed mass-dependence of structure and kinematics (not by effects of stellar population). Our future extensive dynamical studies of galaxy mergers will provide a plausible answer for the problem of whether or not the structural and kinematical nonhomology in merger remnants can really reproduce the $K$ band FP even if stellar populations of galaxy mergers does not contribute at all to the creation of the $K$ band FP.

EDITOR: PLACE FIGURE 11] HERE.

EDITOR: PLACE FIGURE 12 HERE.

EDITOR: PLACE FIGURE 13 HERE. 


\subsection{Miscellaneous results}

In the preceding sections, we have only addressed the importance of initial disk mass $\left(M_{\mathrm{d}}\right)$ and supernova feedback $\left(f_{\mathrm{sn}}\right)$ in determining a number of fundamental mass-dependent properties of elliptical galaxies. Here in $§ 3.3$, we emphasize the importance of parameters other than $M_{\mathrm{d}}$ and $f_{\mathrm{sn}}$ in chemical evolution of gas-rich galaxy mergers. We particularly present the results of models with different chemical mixing length $R_{\text {chem }}$ (Model 10 and Model 11) and total amount of gaseous dissipation (Model 12 and 13). Both chemical mixing length and total amount of gaseous dissipation are less critical for chemical evolution of galaxy mergers than $M_{\mathrm{d}}$ and $f_{\mathrm{sn}}$. It is, however, worth to investigate fundamental roles of these two parameters ( $R_{\text {chem }}$ and effectiveness of gaseous dissipation), since it has not been observationally clarified yet what the most plausible values are for these two parameters. Furthermore we describe mass-independent chemophotometric relations derived in merger remnants.

\subsubsection{Importance of other parameters}

As our Paper I has already pointed out, other merger parameters, such as the mixing length of chemical components and effectiveness of gaseous dissipation during galaxy merging, can greatly determine not only mean properties of stellar populations but also radial ones in merger remnants. The present study, which is more realistic and elaborated than the Paper I, should accordingly confirm the importance of other parameters of galaxy mergers. Figure 11 describes the dependence of the $U-R$ color gradient $(\Delta U-R / \Delta \log R)$ on mean stellar metallicity $\left(\left\langle Z_{*}\right\rangle\right)$ in merger remnants for six models with $M_{\mathrm{d}}=10^{10} M_{\odot}$ and $10^{12} M_{\odot}$ (Model 1, 2, 10, 11, 12, and 13). We include models with chemical mixing length $R_{\text {chem }}=0.1$ (Model 1 and 2), those with $R_{\text {chem }}=0.4$ (Model 10 and 11), and those with $R_{\text {chem }}=0.1$ and no gaseous dissipation (Model 12 and 13) in Figure 11, and thereby 
observe how chemical mixing length $\left(R_{\text {chem }}\right)$ and total amount of gaseous dissipation affect global and radial properties of stellar populations in merger remnants. The importance of chemical mixing length and gaseous dissipation clarified for $\left\langle Z_{*}\right\rangle$ and $\Delta U-R / \Delta \log R$ can be applied to other mean and radial properties of metals, color, and line indices. As is shown in Figure 11, mean stellar metallicity is slightly smaller in the models with larger $R_{\text {chem }}$ (Compare, for example, the results of Model 1 with those of Model 10.), which is consistent with that derived in the Paper I. The $U-R$ color gradient is not largely different between models with different $R_{\text {chem }}$ for less massive models whereas the gradient is appreciably smaller in mergers with larger $R_{\text {chem }}$ for more massive models. This result implies that inhomogeneous chemical mixing described before $(\S 3.1)$ is critical for the formation of color gradient especially for more massive galaxy mergers. Furthermore, mean stellar metallicity is found to be appreciably smaller for models with no gaseous dissipation (Compare, for example, the results of Model 1 with those of Model 12.). This result clearly suggests that total amount of gaseous dissipation during galaxy merging is also important determinant for the mean properties of stellar populations of merger remnants. Figure 11 moreover shows that without gaseous dissipation, the $U-R$ color gradient in merger remnants becomes positive, which suggests that a sufficiently large amount of gaseous dissipation is indispensable for successful reproduction of the observed negative color gradients of luminous elliptical galaxies. These result clearly demonstrate that both chemical mixing length and the degree of gaseous dissipation are important determinants for mean and radial chemical and photometric properties of merger remnants. These results accordingly imply that more careful numerical implementation on chemical mixing and gaseous dissipation in galaxy mergers is required for comparing more quantitatively numerical results with observational ones: The mass-dependent chemical, photometric, and spectroscopic properties of galaxy mergers clarified in the present study (especially in $\S 3.1$ and $§ 3.2)$ can be meaningful only in a qualitative sense. 


\subsubsection{Mass-independent chemophotometric relations}

We have so far restricted ourselves to mass-dependent (or luminosity-dependent) chemical, photometric, and spectroscopic properties of elliptical galaxies. There are, however, a number of observational spectrophotometric properties that correlate not so well with galactic luminosity but strongly with other physical properties in elliptical galaxies. Figure 12 and 13 demonstrate two examples of this type of correlation derived in the present study. Figure 12 shows the dependence of the radial gradient of $\mathrm{Mg}_{2}$ index $\left(\Delta \log \mathrm{Mg}_{2} / \Delta \log R\right)$ on the central $\mathrm{Mg}_{2}$ index (within $0.1 R_{\mathrm{e}}$ ) in merger remnants for all models in the present study. We can clearly observe that merger remnant with larger central $\mathrm{Mg}_{2}$ index has the larger (negative) gradient of $\mathrm{Mg}_{2}$ index. This result is consistent reasonably well with the recent observational result that the radial gradient of $\mathrm{Mg}_{2}$ index can correlate with the central $\mathrm{Mg}_{2}$ index rather than with galactic luminosity or central velocity dispersion (González \& Gorgas 1996). This result furthermore shows that even if there is a great diversity in parameters associated with physical processes of galaxy merging, the observed relationship between the central $\mathrm{Mg}_{2}$ index and $\Delta \mathrm{Mg}_{2} / \Delta \log R$ can be reproduced successfully by galaxy mergers: The origin of $\mathrm{Mg}_{2}-\Delta \mathrm{Mg}_{2} / \Delta \log R$ relation does not depend on the details of galaxy merging processes. Figure 13 gives the dependence of stellar metallicity gradient $(\Delta \log \langle Z\rangle / \Delta \log R)$ on the radial gradient of the epoch of star formation $\left(\Delta \log \left\langle T_{\mathrm{SF}}\right\rangle / \Delta \log R\right)$ in merger remnants for all models in the present study. The radial gradient of $\Delta \log \left\langle T_{\mathrm{SF}}\right\rangle / \Delta \log R$ corresponds to the age gradient in each merger remnant. As is shown in Figure 13, merger remnants with larger absolute magnitude of their age gradients have larger metallicity gradients. This result is consistent with the observational one inferred from the radial gradients of $\mathrm{H}_{\beta}$ and [MgFe] line indices (e.g., Chiosi 1996). The physical reason for the derived dependence is that irrespectively of merger parameters, younger stellar population in the central part of galaxy mergers are formed preferentially from more metal-enriched ISM transferred from the outer part of mergers 
owing to the efficient gas fueling. Thus these two results on the $\mathrm{Mg}_{2}-\Delta \mathrm{Mg}_{2} / \Delta \log R$ relation and $\Delta \log \left\langle T_{\mathrm{SF}}\right\rangle / \Delta \log R-\Delta \log \langle Z\rangle / \Delta \log R$ one provide clues to the origins of observational results of Chiosi (1996) and González \& Gorgas (1996), which clearly show mass-independent properties of elliptical galaxies.

\section{Discussion}

We have demonstrated that spectrophotometric properties of galaxy mergers depend on galactic mass (or luminosity), essentially because galactic mass can greatly affect both the rapidity of star formation and the effectiveness of supernova feedback in chemodynamical evolution of galaxies mergers. This implies that the origins of the observed fundamental characteristics of spectrophotometric properties of elliptical galaxies can be understood in terms of mass-dependent chemodynamical evolution of galaxy mergers. Here we particularly discuss whether or not the present merger model of elliptical galaxy formation can explain quantitatively the origins of the following two fundamental empirical relationship between

galactic mass (luminosity) and spectrophotometric properties in elliptical galaxies: (1) The CM relation and (2) The $\mathrm{Mg}_{2}-\sigma$ one.

\subsection{The origin of the CM relation}

The CM relation is generally considered to be one of important diagnostics which can confirm whether or not a certain model of elliptical galaxy formation is plausible and viable. The present merger model predicts that more massive ellipticals have larger stellar metallicity thus show redder colors. The reasons for more efficient metal-enrichment in more massive galaxy mergers are firstly that a smaller amount of metal-enriched ISM can be tidally stripped away from galaxy mergers owing to more rapid gas consumption by 
star formation, secondly that star formation can be less strongly suppressed by supernova feedback thus can produce a larger amount of metals. These results imply that the origin of the CM relation of elliptical galaxies can be closely associated with mass-dependent chemodynamical evolution of galaxy mergers. Although these results can provide a qualitative explanation of the CM relation, the present merger model still seems to have some difficulties in explaining quantitatively the origin of the absolute magnitude of the CM slope (See Figure 8). To be more specific, the color difference in $V-K$ between a merger remnant with $1.5 \times 10^{9} L_{\odot}$ and that with $1.9 \times 10^{11} L_{\odot}$ in $B$ band is only 0.45 (0.16) mag for models with $f_{\mathrm{sn}}=0.5(0.1)$, which seems to disagree with the observed difference of 0.48 for the corresponding luminosity range in Bower et al. (1992). Since we did not consider any age differences of galaxy mergers with different masses in the present study, this apparent disagreement implies that the present chemodynamical model can not reproduce a mass-metallicity relation, which is one of possible interpretations for the origin of the observed CM relation. There are mainly two interpretations of the CM relation; One is that the CM relation is due only to a mass-metallicity relation, i.e., the relation that more massive (luminous) ellipticals have larger metallicity thus show redder colors, and the other is that both a mass-metallicity relation and a mass-age relation, i.e., the relation that more massive ellipticals are older, play some roles in the formation of the CM relation. We here reject the idea that only a systematical difference in mean galaxy ages between ellipticals with different masses is a key determinant for the CM relation, primarily because some theoretical studies have already demonstrated that this idea is completely inconsistent with the observed redshift evolution of the slopes of the CM relation (e.g., Kodama \& Arimoto 1997). Accordingly, whether or not the apparent disagreement in the successful reproduction of the CM relation is really a serious problem of the present merger model of elliptical galaxy formation depends on which of the above two interpretations we adopt.

Firstly, we adopt the conventional and classical interpretation that elliptical galaxies 
as a whole are formed at high redshift and thus there are not any significant age differences between elliptical galaxies: The origin of the CM relation can be thus understood only in terms of a mass-metallicity relation. In this case, the above apparent disagreement is really a serious problem of the present chemodynamical model of elliptical galaxy formation and thus implies either that more sophisticated modification of the present chemodynamical model is necessary, including dissipative dynamics, SNIa and SNII feedback effects, star formation parameters (e.g, variable IMF), and chemical mixing processes, or that typical luminous elliptical galaxies are not formed by major disk-disk mergers. Secondly, we adopt the interpretation that less luminous ellipticals are as a whole younger and less metal-enriched galaxies: The origin of the CM relation is not necessarily a pure mass-metallicity relation, and thus a systematic age difference between ellipticals can also play a role in the formation of the CM relation (e.g., Faber et al. 1995; Worthey et al. 1996). In this case, the above apparent inconsistency can be resolved by invoking the assumption that less luminous elliptical galaxies are formed by galaxy mergers with initially younger stellar populations or by later galaxy mergers.

The considerably tight color-magnitude relation of elliptical galaxies (Bower, Lucey, \& Ellis 1992; Ellis et al. 1997) and the relatively smaller redshift evolution of photometric properties of elliptical galaxies (Aragón-Salamanca et al. 1993; Franx \& van Dokkum 1996) are generally considered to reflect the coeval formation of elliptical galaxies at high redshift. Furthermore the observed considerably less significant evolution of the slope of the CM relation is suggested to reject the age spread larger thatn 1 Gyr among elliptical galaxies (e.g., Kodama \& Arimoto 1997). These results imply that most of ellipticals are formed at high redshift and thus that the CM relation is a pure mass-metallicity relation. Luminosity-dependence of line index $[\mathrm{MgFe}]$ and $\mathrm{H}_{\beta}$ (Faber et al. 1995 Worthey et al. 1996), on the other hand, indicates that less luminous ellipticals have younger stellar populations. These results imply that a systematic difference in mean galaxy ages between 
ellipticals can play some roles in the formation of the CM relation. Thus, it has not been observationally clarified whether the observed CM relation is due essentially to a pure mass-metallicity relation or both a mass-age relation and a mass-metallicity one contribute to the CM relation. Considering these apparently inconsistent observational results, it is reasonable for the present paper to claim that whether or not the apparent failure of the present merger model implies a serious disadvantage of the merger scenario of elliptical galaxy formation depends on future extensive observational studies on the mass-dependence of mean age and metallicity of elliptical galaxies.

\subsection{Chemodynamical evolution of mergers and the $\mathrm{Mg}_{2}-\sigma$ relation}

The present chemodynamical model predicts that merger remnants with larger central velocity dispersion $(\sigma)$ have larger central $\mathrm{Mg}_{2}$ index, principally because a larger amount of metal-enriched ISM can be transferred to the central region of galaxy mergers and converted into stellar components there in more massive galaxy mergers with larger central velocity dispersion. Although this result can give a qualitative explanation for the origin of $\mathrm{Mg}_{2}-\sigma$ relation of elliptical galaxies, the present merger model has some difficulties in explaining quantitatively the origin (See Figure 9). For models with $f_{\mathrm{sn}}=0.1$, a merger remnant with $\sigma=305 \mathrm{~km} / \mathrm{s}$ has $\mathrm{Mg}_{2}=0.300$ mag whereas that with $\sigma=105 \mathrm{~km} / \mathrm{s}$ has $\mathrm{Mg}_{2}=0.272 \mathrm{mag}$. This result gives the relation $\mathrm{Mg}_{2} \propto 0.06 \log \sigma$. On the other hand, for models with $f_{\mathrm{sn}}=$ 0.5 , a merger remnant with $\sigma=284 \mathrm{~km} / \mathrm{s}$ has $\mathrm{Mg}_{2}=0.291$ mag whereas that with $\sigma=47$ $\mathrm{km} / \mathrm{s}$ has $\mathrm{Mg}_{2}=0.177 \mathrm{mag}$. This result gives the relation $\mathrm{Mg}_{2} \propto 0.16 \log \sigma$. The derived $\mathrm{Mg}_{2}-\sigma$ relations for the above two cases $\left(f_{\mathrm{sn}}=0.1\right.$ and 0.5$)$ are not consistent reasonably

well with the observed relation $\mathrm{Mg}_{2} \propto 0.2 \log \sigma$ (Burstein et al. 1988; Davies 1996; Jøgensen 1997). The reason for this inconsistency is primarily that the absolute magnitude of $\mathrm{Mg}_{2}$ for less luminous merger remnants in the present study, in particular, for models with $f_{\mathrm{sn}}$ 
$=0.0$ and 0.1 , is appreciably larger than the observed one. We did not consider any age differences of galaxy mergers with different masses in the present study. Accordingly this inconsistency implies that the present chemodynamical model have failed to reproduce a metallicity- $\sigma$ relation, which is one of possible interpretations for the origin of the observed $\mathrm{Mg}_{2}-\sigma$ relation. The observed small scatter in the $\mathrm{Mg}_{2}-\sigma$ relation (Bender, Burstein, \& Faber 1993) probably allows the following two interpretations on the origin of the $\mathrm{Mg}_{2}-\sigma$ relation. One is that a $\mathrm{Mg}_{2}-\sigma$ relation results exclusively from a metallicity- $\sigma$ relation (i.e., the relation that a larger $\sigma$ galaxy has larger stellar metallicity), and the other is that both a metallicity- $\sigma$ relation and a age- $\sigma$ (i.e., the relation that a larger $\sigma$ galaxy has older stellar age) can contribute to the $\mathrm{Mg}_{2}-\sigma$ relation (We here consider that the observed small scatter in the $\mathrm{Mg}_{2}-\sigma$ relation rules out the interpretation that only a systematic age difference between ellipticals can explain totally the origin of the $\mathrm{Mg}_{2}-\sigma$ relation). Accordingly, whether or not our failure in the successful reproduction of the $\mathrm{Mg}_{2}-\sigma$ relation is a serious problem of the present merger model of elliptical galaxy formation depends on which of the above two interpretations we adopt.

Firstly, we adopt the interpretation that the $\mathrm{Mg}_{2}-\sigma$ relation reflects only the dependence of stellar mean metallicity on $\sigma$ (or mass) in the central part of elliptical galaxies. In this case, the $\mathrm{Mg}_{2}-\sigma$ relation derived in the present merger model of elliptical galaxies is really inconsistent with observational one, which implies that inward transfer of metals $(\mathrm{Mg})$ during dissipative galaxy merging is not so efficient as the present merger model predicts especially for less luminous galaxy mergers. For this case, we must either construct a more elaborated and realistic chemodynamical model of galaxy mergers and thereby explore again the origin of the $\mathrm{Mg}_{2}-\sigma$ relation or conclude that major disk-disk galaxy merging is not closely associated with the formation of typical elliptical galaxies. Secondly, we adopt the interpretation that less luminous ellipticals with smaller $\sigma$ are more likely to show both smaller metallicity and younger ages thus show smaller $\mathrm{Mg}_{2}$. In this case, our failure is not 
so serious problem for the present chemodynamical model of elliptical galaxy formation, principally because we can reproduce the $\mathrm{Mg}_{2}-\sigma$ relation by invoking the assumption that less luminous ellipticals with smaller $\sigma$ are formed by mergers with initially younger stellar populations or by later mergers.

As is the case of the CM relation, it is observationally unclear which of the above two interpretations is more plausible and reasonable for the origin of the $\mathrm{Mg}_{2}-\sigma$ relation. Observation studies on the redshift evolution of the $\mathrm{Mg}_{2}-\sigma$ relation in cluster ellipticals (Ziegler \& Bender 1997), the color-magnitude diagram of stellar populations of M32 by HST (Grillmair et al. 1996), and the dependence of the slope of the FP on the observational wavelength (Pahre et al. 1998) indicate younger stellar populations in less luminous ellipticals. The considerably smaller scatter in the $\mathrm{Mg}_{2}-\sigma$ relation, however, implies coeval formation of elliptical galaxies at higher redshift universe (Bender et al. 1993). Furthermore there are no observational studies, at least now, which clearly demonstrate that less luminous ellipticals as a whole have younger stellar populations in the central part of galaxies. We are accordingly conservative to determine the more plausible interpretation for the $\mathrm{Mg}_{2}-\sigma$ relation. Thus, it is safe for us to say that it depends on more extensive future observational studies whether or not the present numerical results on the $\mathrm{Mg}_{2}-\sigma$ relation are really consistent with observational ones.

\section{Conclusion}

We have numerically investigated chemodynamical evolution of major disk-disk galaxy mergers in order to explore the origin of mass-dependent chemical, photometric, and spectroscopic properties observed in elliptical galaxies. The present study is an extended version of our previous study (Paper I), which is the first step toward the understanding of a close physical relationship between dynamical evolution and chemical one in galaxy mergers. 
The present chemodynamical model is more realistic and sophisticated than our Paper I in the following three points: (1) Instead using instantaneous chemical mixing approximation, we consider time-delay between star formation and metal ejection mainly from Type Ia and II supernovae. (2) Feedback effects of Type Ia and II supernovae on dynamical and chemical evolution of galaxy mergers are incorporated. (3) Time-evolution of enrichment processes is solved for each of chemical components, $\mathrm{H}, \mathrm{He}, \mathrm{Mg}, \mathrm{O}$, and Fe. We have found that initial galactic mass $\left(M_{\mathrm{d}}\right)$ is one of critical determinants for chemical, photometric, and spectroscopic properties of merger remnants. The essential reason for the derived mass-dependence is that galactic mass can largely determine total amount of metal-enriched interstellar gas, star formation histories of galaxy mergers, and the effectiveness of Type Ia and II supernova feedback, all of which greatly affect chemodynamical evolution of galaxy mergers. In particular, the difference in the effectiveness of Type Ia and II supernova feedback between galaxy mergers with different masses is found to determine not only the mean properties but also the radial ones in ellipticals with different masses.

What we should emphasize here is that since this paper is only the first step toward the deep understanding of chemodynamical and spectrophotometric evolution of galaxy mergers, we completely neglect some possibly important effects of galactic dynamics on chemophotometric evolution of galaxies. Accordingly, some dynamical effects that are not investigated in the present paper can greatly modify the derived mass-dependence of chemophotometric properties of merger remnants. Among these, dynamical effects of galactic bulges are particularly important in the sense that bulges can largely control inward and radial mass-transfer of interstellar gas (Mihos \& Hernquist 1996) and consequently determine star formation histories of galaxy mergers and the nature of stellar populations formed by the induced secondary starbursts. Probably these dynamical effects of bulges modify the absolute magnitude of radial gradients of spectrophotometric properties derived in the present merger model without bulges. Our future studies with a more realistic merger 
model will investigate in detail how galaxy bulges affect the nature of stellar populations of merger remnants.

Main results obtained in this study are the following seven.

(1) More massive (luminous) ellipticals formed by galaxy mergers between more massive spirals have larger metallicity and thus show redder colors, principally because a larger amount of metal-enriched ISM can be converted into stellar components in more massive galaxy mergers. This result implies that the origin of the CM relation of elliptical galaxies can be closely associated with dissipative galaxy merging with star formation. The typical metallicity ranges from $\sim 1.0$ solar abundance $(Z \sim 0.02)$ for ellipticals formed by mergers with $M_{\mathrm{d}}=10^{10} M_{\odot}$ to $\sim 2.0$ solar $(\mathrm{Z} \sim 0.04)$ for those with $M_{\mathrm{d}}=10^{12} M_{\odot}$.

(2) The absolute magnitude of negative metallicity gradients developed in galaxy mergers is more likely to be larger for massive ellipticals. Typical value of metallicity gradient $\left(\Delta \log \left\langle Z_{*}\right\rangle / \Delta \log R\right.$ ) for $0.1 \leq R / R_{\mathrm{e}} \leq 1.0$ (where $R_{\mathrm{e}}$ is effective radius of a merger remnant) is -0.13 for ellipticals with $M_{\mathrm{d}}=10^{12} M_{\odot}$ and -0.03 for those with $M_{\mathrm{d}}=10^{10} M_{\odot}$. Absolute magnitude of metallicity gradient correlates with that of age gradient in ellipticals in the sense that an elliptical with steeper negative metallicity gradient is more likely to show steeper age gradient.

(3) Irrespective of galactic mass, a stellar population in the central part is both younger and more metal-enriched than that in the outer part in an elliptical galaxy formed by dissipative galaxy merging with star formation. This is essentially because metal-enriched ISM can be transferred to the central part of galaxy mergers owing to efficient gas fueling during dissipative galaxy merging with star formation and preferentially converted into stellar components there in the later phase of the merging.

(4) Abundance ratio of $[\mathrm{Mg} / \mathrm{Fe}]$ does not depend so strongly on galactic mass (typically 
$0.2 \sim 0.3$ solar $)$. The absolute magnitude of the radial gradient of $[\mathrm{Mg} / \mathrm{Fe}]$ is rather small and does not depend on galactic mass $\left(M_{\mathrm{d}}\right)$.

(5) Radial color gradient is more likely to be larger for more massive ellipticals, which reflects that the metallicity gradient is larger for more massive ellipticals. For example, typical $U-R$ color gradient $(\Delta U-R / \Delta \log R)$ for $0.1 \leq R / R_{\mathrm{e}} \leq 1.0$ is -0.13 for ellipticals with $M_{\mathrm{d}}=10^{12} M_{\odot}$ and -0.07 for those with $M_{\mathrm{d}}=10^{10} M_{\odot}$.

(6) $\mathrm{Mg}_{2}$ line index in the central part of ellipticals $\left(R \leq 0.1 R_{\mathrm{e}}\right)$ is larger for more massive ellipticals, principally because a larger amount of metal-enriched ISM can be fueled to the central part of mergers and converted into stellar components there in more massive galaxy mergers. This result implies that the origin of $\mathrm{Mg}_{2}-\sigma$ relation observed in elliptical galaxies can be closely associated with the difference in total amount of metal-enriched ISM transferred to galactic center between galaxy mergers with different masses. The radial gradient of $\mathrm{Mg}_{2}\left(\Delta \mathrm{Mg}_{2} / \Delta \log \mathrm{R}\right)$ is also more likely to be larger for massive ellipticals. $\Delta \mathrm{Mg}_{2} / \Delta \log \mathrm{R}$ correlates well with the central $\mathrm{Mg}_{2}$ in ellipticals. For most of the present merger models, ellipticals show positive radial gradient of $\mathrm{H}_{\beta}$ line index.

(7) Both $M / L_{B}$ and $M / L_{K}$, where $M, L_{B}$, and $L_{K}$ are total stellar mass of galaxy mergers, $B$-band luminosity, and $K$-band one, respectively, depend on galactic luminosity in such a way that more luminous ellipticals have larger $M / L_{B}$ and smaller $M / L_{K}$. This result reflects that more luminous merger remnants are more likely to have larger mean stellar metallicity.

These seven numerical results derived in the present chemodynamical merger model of elliptical galaxy formation imply that the observed fundamental dependence of chemophotometric properties of ellipticals on galactic mass or luminosity (e.g., the CM relation, the $\mathrm{Mg}_{2}-\sigma$ relation, and the FP) can be understood in terms of mass-dependent chemodynamical evolution of galaxy merges. 
K.B. thanks to the Japan Society for Promotion of Science (JSPS) Research Fellowships for Young Scientist. 


\section{A. Chemical, photometric, and spectroscopic evolution of SSP with variously different IMF and $M_{\text {low }}$}

Chemical, photometric, and spectroscopic evolution of galaxies are generally considered to depend not only on star formation histories of galaxies resulting from dynamical evolution of galaxies, as is investigated in the present study, but also on detailed properties of IMF, $M_{\text {low }}$, and $M_{\mathrm{up}}$. Accordingly we should emphasize that the results derived in the present study (with the Salpeter IMF, $M_{\text {low }}=0.6 M_{\odot}$, and $M_{\text {up }}=120.0 M_{\odot}$ ) are only true for galaxy mergers with the Salpeter IMF, $M_{\text {low }}=0.6 M_{\odot}$, and $M_{\mathrm{up}}=120.0 M_{\odot}$ in a quantitative sense: We should admit limited applicability of the present chemodynamical model. We here present dependence of chemical, photometric, and spectroscopic evolution of a singe stellar population (SSP) on IMF, $M_{\text {low }}$, and $M_{\text {up }}$, and thereby show more clearly how strongly or weakly the results derived in the present numerical results can depend on IMF, $M_{\text {low }}$, and $M_{\text {up }}$. In particular, we describe (1) Time evolution of ejected metals in a SSP with variously different IMF and $M_{\text {low }}$, and (2) Photometric and spectroscopic evolution of the SSP. These two descriptions will help us to understand how numerical results derived in the present study can be changed if we adopt IMF, $M_{\text {low }}$, and $M_{\text {up }}$ other than those adopted in the present study.

\section{A.1. Time evolution of stellar yield $\left(Y_{i, j}(t)\right)$}

Time evolution of total amount of metals $\left(\left(Y_{i, j}(t)\right)\right.$ ejected from stars with variously deferent mass and lifetime in a SSP with a given metallicity depends on IMF and $M_{\text {low }}$. We here observe how the time evolution of $\mathrm{O}, \mathrm{Fe}, \mathrm{Mg}$, and $\mathrm{Z}$ ejected from stars depends on IMF and $M_{\text {low }}$, by showing the results for models with the Salpeter IMF, $M_{\text {low }}=0.1 M_{\odot}$, and $M_{\text {up }}=120.0 M_{\odot}\left(\right.$ Model A), with the Salpeter IMF, $M_{\text {low }}=0.6 M_{\odot}$, and $M_{\text {up }}=120.0 M_{\odot}$ (Model B), and with IMF with shallower slope (the exponent of IMF equal to -1.1), 
$M_{\text {low }}=0.1 M_{\odot}$, and $M_{\text {up }}=120.0 M_{\odot}($ Model C $)$. The time evolution of Model B corresponds to $Y_{i, j}(t)$ adopted in the main manuscript. It is clear from Figure 14 that (1) Z is larger in a SSP with larger $M_{\text {low }}$ for a given IMF, (2) Z is larger in a SSP with shallower IMF slope for a given $M_{\text {low }}$, and (3) The results of (1) and (2) can be applied to O, Mg, and Fe. These three results, though based on SSP analysis, are well consistent with previous studies solving fully chemical evolution of closed-box one-zone galactic models (Arimoto \& Yoshii 1987; Vazdekis et al. 1996). These results accordingly imply that the absolute magnitude of present numerical results are changed if we adopt IMF and $M_{\text {low }}$ other than those adopted in the present study. For example, if we adopt smaller $M_{\text {low }}$ (shallower IMF slope), mean stellar metallicity of galaxy mergers can become substantially smaller (larger). Thus, we again stress that although the relative importance of each parameter (e.g., galactic mass) in chemophotometric evolution of galaxy mergers derived in the present study does not depend on IMF and $M_{\text {low }}$, the derived absolute magnitude of chemophotometric properties in merger remnants should be more carefully interpreted owing to dependences of chemical properties on IMF and $M_{\text {low }}$ derived in the above three results.

In order to assess the validity of $Y_{i, j}(t)$ adopted in the present study, we furthermore check whether or not the time-evolution of stellar yield $\left(Y_{i, j}(t)\right)$ adopted in the present study can reproduce reasonably well even the chemical evolution of the abundance ratio of $[\mathrm{Mg} / \mathrm{Fe}]$, which has been already investigated by classical one-zone chemical evolution models (e.g., Matteucci \& Tornambè 1987). We investigate the long-term evolution ( 10 Gyr) of stellar $[\mathrm{Mg} / \mathrm{Fe}]$ (the ratio of stellar $\mathrm{Mg}$ abundance to stellar Fe one in units of solar $[\mathrm{Mg} / \mathrm{Fe}])$ produced by stellar systems with three different types of SSP in Model A, B, and C. We check whether or not the $[\mathrm{Mg} / \mathrm{Fe}]$ can finally be 0.0 that is a typical value for the solar neighborhood in the Galaxy, in which chemical evolution is considered to proceed for more than 10 Gyr. Figure 15 describes the time evolution of $[\mathrm{Mg} / \mathrm{Fe}]$ for Model A, B, and C. It is clear from Figure 15 that (1) Model B, the $Y_{i, j}(t)$ of which is exactly the same as 
that used in the main manuscript, can reproduce the typical value of $[\mathrm{Mg} / \mathrm{Fe}]$ in the solar neighborhood in the Galaxy $([\mathrm{Mg} / \mathrm{Fe}] \sim 0.0)$ after long-term evolution, and (2) Final value of $[\mathrm{Mg} / \mathrm{Fe}]$ does not depend so strongly on IMF and $M_{\text {low }}$. These results accordingly justify the $Y_{i, j}(t)$ adopted in the present study. Considering the $[\mathrm{Mg} / \mathrm{Fe}]$ value of $0.2 \sim 0.3$ derived for galaxy mergers and that of $\sim 0.0$ for the above SSP models with long-term and less rapid chemical evolution, the above two results furthermore imply that the higher values of $[\mathrm{Mg} / \mathrm{Fe}]$ derived in the present merger model is closely associated with dissipative galaxy merging with rapid star formation.

EDITOR: PLACE FIGURE 14 HERE.

EDITOR: PLACE FIGURE 15 HERE.

EDITOR: PLACE FIGURE 16 HERE.

\section{A.2. Time evolution of spectrophotometric properties in SSP}

We show how fundamental characteristics of spectrophotometric evolution of SSP with solar metallicity depend on IMF and $M_{\text {low }}$ in Figure 16 for Model A, B, and C. In calculating spectrophotometric properties of SSP, we adopt the PEGASE code of Fioc \& Rocca-Volmerange (1997) which can reproduce the GISSEL96 SSP. It is clear from Figure 16 that (1) The ratio of stellar mass $(M)$ to luminosity $\left(L_{\mathrm{B}}\right)$ is strongly dependent on IMF and $M_{\text {low }}$, and (2) The $B-V$ and $V-K$ colors are not so different between models with different IMF and $M_{\text {low }}$. In the present study, we derive spectrophotometric properties of merger remnant by using the GISSEL96 SSP in which the Salpeter IMF and $M_{\text {low }}=0.1 M_{\odot}$ 
are assumed, nevertheless we adopt the Salpeter IMF and $M_{\text {low }}=0.6 M_{\odot}$ (That is, we adopt a certain 'approximation' in deriving the spectrophotometric properties.). Accordingly, spectrophotometric evolution of galaxy mergers is not self-consistently solved in a strict sense and more careful interpretations for results derived in the present study are necessary. Observing the above two results on the differences in spectrophotometric properties of SSP between Model A $\sim \mathrm{C}$, we can assess the validity of the 'approximation': Global integrated colors derived in the present study can be approximately correct whereas mass-to-light-ratio can not. Thus we must again emphasize that discussing the dependence of spectrophotometric properties of merger remnants on galactic parameters such as initial disk mass is considerably sensible whereas comparing the absolute magnitude of the present numerical results (in particular, mass-to-light-ratio) with those of observational ones is not plausible: What is more vital in the present study is to clarify the relative importance of galactic parameters (e.g., galactic mass) in determining spectrophotometric properties of merger remnants.

\section{B. A possible luminosity dependence of $C_{\mathrm{SF}}$ and $f_{\mathrm{eff}}$}

The essential reason for mass-dependent properties of galaxy mergers is that galactic mass can determine both $C_{\mathrm{SF}}$ and $f_{\mathrm{eff}}$. We here describe the reason for the mass-dependence of $C_{\mathrm{SF}}$ and $f_{\text {eff }}$. Observational background for the mass-dependence of $C_{\mathrm{SF}}$ is given in detail in our Paper I. Firstly the expected mass dependence of $C_{\mathrm{SF}}$ is described as follows. The parameter $C_{\mathrm{SF}}$ is set to be proportional to $T_{\mathrm{dyn}} / T_{\mathrm{SF}}$, where $T_{\mathrm{dyn}}$ and $T_{\mathrm{SF}}$ are the dynamical time-scale and the time-scale of gas consumption by star formation, respectively. We here

define the mass of a galactic disk, the total mass of luminous and dark matter, and size of the progenitor as $M_{d}, M_{t}$, and $R_{d}$, respectively. We consider here that gas mass in a disk is 
equal to $M_{d}$ for simplicity. The $T_{\text {dyn }}$ is given as

$$
T_{\mathrm{dyn}} \propto R_{d}^{3 / 2} M_{t}^{-1 / 2}
$$

Provided that the coefficient in the Schmidt law is not dependent on the galactic mass (or luminosity), we can derive $T_{\mathrm{SF}}$ as follows.

$$
T_{\mathrm{SF}} \propto \Sigma^{1-\gamma}
$$

where $\Sigma$ is the surface density of the gas disk. The parameter $\gamma$ is the exponent of Schmidt law, which is the same as that used in previous subsections. Assuming the Freeman's law and the constant ratio of $R_{d}$ to the scale length of exponential disk, we derive

$$
\Sigma \propto M_{d} R_{d}^{-2} \sim \text { const. }
$$

Assuming that the degree of self-gravity of a galactic disk is described as

$$
M_{t} \propto M_{d}^{(1-\beta)}
$$

then we can derive

$$
C_{\mathrm{SF}} \propto M_{d}^{1 / 4+\beta / 2}
$$

Since $\beta$ is considered to have positive value $(\beta=0.6$ : Saglia 1996), this relationship predicts that $C_{\mathrm{SF}}$ becomes larger as $M_{d}$ increases. If we adopt the observed trend that more luminous disks have larger surface density, such as $\Sigma \propto M_{d}$ (McGaugh \& Blok 1997), instead of using the Freeman's law, we can obtain $C_{\mathrm{SF}} \propto M_{d}^{1 / 2+\beta / 2}$. Since in the present study, we assume, for simplicity, that the ratio of dynamical mass to initial disk mass is constant for galaxy mergers with different masses (that is, $\beta=0.0$ ), and furthermore that $M_{\mathrm{d}}$ is proportional to $R_{d}^{2}$ (Freeman's law), the $M_{\mathrm{d}}$ dependence of $C_{\mathrm{SF}}$ adopted in the present study is described as:

$$
C_{\mathrm{SF}} \propto M_{d}^{0.25}
$$


Secondly the expected mass dependence of $f_{\text {eff }}$ is described as follows. If we neglect the importance of details of three dimensional structure of a galaxy with dark matter halo in determining the total potential energy of the galaxy, total potential energy of an initial disk $\left(W_{\mathrm{d}}\right)$ is then considered to be within an order of $G M_{\mathrm{t}}^{2} / R_{\mathrm{d}}$. Assuming that the total number of supernovae is basically dependent on $M_{\mathrm{d}}$, the total energy of supernovae that can be potentially accumulated within the disk $\left(E_{\mathrm{SN}}\right)$ is proportional to $e_{\mathrm{sn}} M_{\mathrm{d}}$, where $e_{\mathrm{sn}}$ is total energy produced by one supernova $\left(\sim 10^{51}\right.$ erg both for SNIa and SNII). Accordingly, the ratio of total supernova energy (that can be ejected in the course of galactic evolution) to total potential energy of the galaxy, which corresponds to the effectiveness of supernova feedback in galactic evolution, is as follows:

$$
f_{\mathrm{eff}} \propto E_{\mathrm{SN}} / W_{d} \propto e_{\mathrm{sn}} M_{\mathrm{d}} / G M_{\mathrm{t}}^{2} / R_{\mathrm{d}} \propto R_{\mathrm{d}} / M_{\mathrm{d}} \propto M_{\mathrm{d}}^{-0.5}
$$

In the above equation, the constant $M_{\mathrm{t}} / M_{\mathrm{d}}$ and $M_{\mathrm{d}} \propto R_{d}^{2}$ are assumed. This equation means that supernova feedback effects can not so strongly affect evolution of galaxies for more massive galaxy mergers. It should be emphasized here that the expected $M_{\mathrm{d}}$ dependences of $C_{\mathrm{SF}}$ and $f_{\text {eff }}$ are based on some assumptions adopted for deriving these dependence. 


\section{REFERENCES}

Aragón-Salamanca, A., Ellis, R. S., Couch, W. J., \& Carter D. 1993, MNRAS, 262, 764

Arimoto, N., \& Yoshii, Y. 1987, A\&A, 173, 23

Barnes, J., \& Hernquist, L. 1992, ARA\&A, 30, 705

Bekki, K. 1998, ApJ, 496, 713

Bekki, K., \& Shioya, Y. 1997, ApJ, 478, L17

Bekki, K., \& Shioya, Y. 1998, ApJ, 497, 108 (Paper I)

Bender, R., Burstein, D., \& Faber S. M. 1992, ApJ, 399, 462

Bender, R., Burstein, D., \& Faber S. M. 1993, ApJ, 411, 153

Binney, J., \& Tremaine, S. 1987 in Galactic Dynamics, Princeton; Princeton Univ. Press.

Bower, R. G., Lucey, J. R., \& Ellis, R. S. 1992, MNRAS, 254, 601

Bressan, A., Fagotto, F., Bertelli, G., \& Chiosi, C. 1993, A\&AS, 100, 647

Bressan, A., Chiosi C., \& Tantalo, R. 1996, A\&A, 311, 425

Bruzual A., G., \& Charlot, S. 1993, ApJ, 405, 538

Burstein, D., Bertola, F., Buson, L. M., Faber, S. M., \& Lauer, T. R. 1988, ApJ, 328, 440

Capelato, H. V., de Carvalho, R. R., \& Carlberg R. G. 1995, ApJ, 451, 525

Carlberg, R. G. 1984, ApJ, 286, 416

Chiosi, C. in Fresh Views of Elliptical Galaxies, ed. A. Buzzoni, R. Renzini, and A. Serrano, ASP Conf. Ser. Vol 86, p215 
Davies, R. L., Sadler, E. M., \& Peletier, F. 1993, MNRAS, 262, 650

Davies, R. L., in Fresh Views of Elliptical Galaxies, ed. A. Buzzoni, R. Renzini, and A. Serrano, ASP Conf. Ser. Vol 86, p175

Djorgovski, S., \& Davis, M. 1987, ApJ, 313, 59

Djorgovski, S., Pahre, M. A., \& de Carvalho R. R. 1996, in Fresh Views of Elliptical Galaxies, ed. A. Buzzoni, R. Renzini, and A. Serrano, ASP Conf. Ser. Vol 86, p129

Dressler, A., Lynden-Bell, D., Burstein, D., Davies, R. L., Faber, S. M., Terlevich, R. J., \& Wegner, G. 1987, ApJ, 313, 42

Ellis, R. S., Smail, I., Dressler, A., Couch, W. J., Oemler, A., Butcher, H., \& Sharples R.M. 1997, ApJ, 483, 582

Faber, S. M. 1973, ApJ, 179, 731

Faber, S. M., Trager, S. C., Gonzalez, J. J., \& Worthey, G. 1995, in Stellar Population ed. P.C. van der Kruit and G.Gilmore, IAU 164, p. 249

Fall, S. M., \& Efstathiou, G. 1980, MNRAS, 193, 189

Fioc, M., \& Rocca-Volmerange, B. 1997, A\&A, 326, 950

Franx, M. \& van Dokkum, P. G. 1996, in New light on galaxy evolution ed. R. Bender, R. Davies, IAU symp. 171, p233

Freeman, K. C. 1970, ApJ, 160, 811

Fritze-v. Alvensleben, U., \& Gerhard, O. E. 1994, A\&A, 285, 751

Gibson, B. K., \& Matteucci, F. 1997, ApJ, 475, 47 
González, J. J., \& Gorgas, J. 1996, in Fresh Views of Elliptical Galaxies, ed. A.Buzzoni, A.Renzini, and A.Serrano, ASP Conf.Ser. Vol.86, p. 225

Grillmair, C. J. et al. 1996, AJ, 112, 1975

Jørgensen, I. 1997, MNRAS, 288, 161

Katz, N. 1992, ApJ, 391, 502

Kauffmann, G., \& Charlot, S. 1997, preprint astro-ph/9704148

Kennicutt, R. C. 1983, ApJ, 272, 54

Kennicutt, R. C. 1989, ApJ, 344, 685

Kodama, T., \& Arimoto, N. 1997, A\&A, 320, 41

Larson, R. B. 1972, Nat, 236, 21

Larson, R. B. 1975, MNRAS, 173, 671

Larson, R. B., Tinsley, B. M., \& Caldwell, C. N. 1980, ApJ, 237, 692

Larson, R. B. 1992, in Star formation in stellar systems, ed. G. Tenorio-Tagle, M. Prieto, and F. Sánchez, p 127

Magris, G., \& Bruzual A., G. 1993, ApJ, 417, 102

Matteucci, F., \& Tornambè, A. 1987, A\&A, 185, 51

Matteucci, F., \& Franoiis, P. 1989, MNRAS, 239, 885

McGaugh, S. S., \& de Blok, W. J. G. 1997, ApJ, in press

McKee, C. F., \& Ostriker, J. P. 1977, ApJ, 218, 148 
Mihos, J. C., Richstone, D. O., \& Bothun, G. D. 1992, ApJ, 400, 153

Mihos, J. C., \& Hernquist, L. 1996, ApJ, 464, 641

Navarro, J., \& White, S. D. M. 1993, MNRAS, 265, 271

Nomoto, K., Thielemann, F.-K., \& Yokoi, K. 1984, ApJ, 286, 644

Pahre, M. A., \& Djorgovski, S. G. 1997, in ASP Conf. Ser. 116, The second Stromlo symposium: The nature of elliptical galaxies, edited by M. Arnaboldi, G. S. Da Costa, \& P. Saha, 154

Pahre, M. A., de Carvalho, R. R., \& Djorgovski, S. G. 1998, AJ, in press (astro-ph/9806326)

Peletier, R. F., Valentijn, E. A., \& Jameson, R. F. 1990a, A\&A, 233, 62

Peletier, R. F., Davies, R. L., Illingworth, G. D., Davis, L. E., \& Cawson, M. 1990b, AJ, 100, 1091

Saglia, R. P. 1996, in New light on galaxy evolution ed. R. Bender, R. Davies, IAU symp. $171, \mathrm{p} 157$

Sandage, A. 1986, A\&A, 161, 89

Schmidt, M. 1959, ApJ, 344, 685

Schwarz, M. P. 1981, ApJ, 247, 77

Schweizer, F. \& Seitzer, P. 1992, AJ, 104, 1039

Sugimoto, D., Chikada, Y., Makino, J., Ito, T., Ebisuzaki, T., \& Umemura, M. 1990, Nature, 345, 33

Tantalo, R., Chiosi, C., Bressan, A., \& Fagotto, F. 1996, A\&A, 311, 361 
Toomre, A., \& Toomre, J. 1972, ApJ, 178,623

van den Bergh, S. 1962, AJ, 67, 486

Vazdekis, A., Casuso, E., Peletier, R. F., \& Beckman, J. E., 1996, ApJS, 106, 307

Visvanathan, N., \& Sandage, A. 1977, ApJ, 216, 214

White, S. D. M. 1980, MNRAS, 191, 1p

White, S. D. M., \& Rees, M. J. 1978, MNRAS, 183, 341

Wielen, R. 1977, A\&A, 60, 263

Woosley, S. E., \& Weaver, T. A. 1995, ApJS, 101, 181

Worthey, G., Trager, S. C., \& Faber, S. M. 1996, in Fresh Views of Elliptical Galaxies, ed. A.Buzzoni, A.Renzini, and A.Serrano, ASP Conf.Ser. Vol.86, p. 203

Ziegler, B., \& Bender, R. 1997, MNRAS, 291, 527 
Fig. 1. - Time evolution of gas mass fraction $\left(f_{\mathrm{g}}\right)$ and mean stellar metallicity $\left(\left\langle Z_{*}\right\rangle\right)$ for models with $M_{\mathrm{d}}=10^{10} M_{\odot}$ (Model 1 , solid lines) and $M_{\mathrm{d}}=10^{12} M_{\odot}$ (Model 2, dotted ones). For the two models, the time evolution for 16 dynamical time $\left(t_{\mathrm{dyn}}\right)$ of each galaxy merger is described. Note that both stellar mass fraction and mean stellar metallicity are larger for the more massive galaxy merger.

Fig. 2.- Final metallicity distribution of stellar component $\left(Z_{*}\right)$ in merger remnants at $T=16 t_{\mathrm{dyn}}$ for models with $M_{\mathrm{d}}=10^{10} M_{\odot}$ (Model 1 , shading bars) and $M_{\mathrm{d}}=10^{12} M_{\odot}$ (Model 2, open ones). The time $T=16 t_{\text {dyn }}$ corresponds to $\sim 1.4$ Gyr for Model 1 and $\sim 4.6$ Gyr for Model 2. Fractional mass of stellar components with a given metallicity (in $\log Z_{*}$ ) is shown for each metallicity bin. Note that the more massive galaxy merger has a larger amount of substantially metal-enriched stellar populations.

Fig. 3.- Time evolution of mean stellar metallicity of $\left\langle Z_{*, F e}\right\rangle$ (top) and $\left\langle Z_{*, M g}\right\rangle$ (middle) and that of the abundance ratio of $[\mathrm{Mg} / \mathrm{Fe}]$ (bottom) in merger remnants for models with $M_{\mathrm{d}}=10^{10} M_{\odot}\left(\right.$ Model 1 , solid lines) and $M_{\mathrm{d}}=10^{12} M_{\odot}$ (Model 2, dotted ones). For the two models, the time evolution for 16 dynamical time of each galaxy merger is described.

Fig. 4. - Radial profiles of metallicity gradients for $\left\langle Z_{*}\right\rangle$ (top), $\left\langle Z_{*, F e}\right\rangle$ (second from the top), and $\left\langle Z_{*, M g}\right\rangle$ (second from the bottom) and that of $[\mathrm{Mg} / \mathrm{Fe}]$ in merger remnants at $T=16 t_{\mathrm{dyn}}$ for models with $M_{\mathrm{d}}=10^{10} M_{\odot}$ (Model 1 , solid lines) and with $M_{\mathrm{d}}=10^{12} M_{\odot}$ (Model 2, dotted ones). The time $T=16 t_{\text {dyn }}$ corresponds to $\sim 1.4$ Gyr for Model 1 and $\sim$ 4.6 Gyr for Model 2.

Fig. 5. - Radial profiles of mean epoch of star formation $\left(\left\langle T_{\mathrm{SF}}\right\rangle\right)$ in merger remnants at $T=16 t_{\mathrm{dyn}}$ for models with $M_{\mathrm{d}}=10^{10} M_{\odot}$ (Model 1 , solid lines) and with $M_{\mathrm{d}}=10^{12} M_{\odot}$ (Model 2, dotted ones). The larger value of $\left\langle T_{\mathrm{SF}}\right\rangle$ means younger stellar populations in each radius. The time $T=16 t_{\text {dyn }}$ corresponds to $\sim 1.4$ Gyr for Model 1 and $\sim 4.6$ Gyr for Model 
2. Note that irrespectively of mass of galaxy mergers, stellar populations in the central part of merger remnants are younger than those in the outer part of merger remnants.

Fig. 6. - Radial profiles of color gradients for $U-R$ (top), $B-R$ (middle) and $V-K$ (bottom) in merger remnants at the age of 13.1 Gyr for models with $M_{\mathrm{d}}=10^{10} M_{\odot}$ (Model 1, solid lines) and with $M_{\mathrm{d}}=10^{12} M_{\odot}$ (Model 2, dotted ones). The rapid increase and decrease of colors within $0.1 R_{\mathrm{e}}$ are due to the smaller particle number in the central part of each merger remnant. For comparison, the observed mean color gradients (Peletier et al. 1990a, b) with arbitrary zero-points are given by dash-dotted lines. Here mean values of color gradients for $U-R$ (top), $B-R$ (middle), and $V-K$ (bottom) are $-0.20,-0.09$, and -0.14 , respectively.

Fig. 7.- Radial profiles of line indices for $\mathrm{H}_{\beta}$ (top), $\mathrm{Mg}_{2}$ (middle) and $\langle F e\rangle$ (bottom) in merger remnants at the age of 13.1 Gyr for models with $M_{\mathrm{d}}=10^{10} M_{\odot}$ (Model 1 , solid lines) and with $M_{\mathrm{d}}=10^{12} M_{\odot}$ (Model 2, dotted ones). The rapid increase and decrease of line indices within $0.1 R_{\mathrm{e}}$ are due to the smaller particle number in the central part of each merger remnant. For comparison, the observed mean $\mathrm{Mg}_{2}$ gradient (Davies, Sadler, \& Peletier 1993) with arbitrary zero-points are given by dash-dotted lines. Here mean values of line index gradients for $\mathrm{H}_{\beta}$ (top), $\mathrm{Mg}_{2}$ (middle), and $\langle F e\rangle$ (bottom) are 0.23, -0.059, and -0.38 , respectively.

Fig. 8. - The luminosity $\left(L_{B}\right.$ in units of $L_{\odot}$ ) dependence of $U-R$ (top), $B-R$ (middle), and $V-K$ color (bottom) in merger remnants at the age of $13.1 \mathrm{Gyr}$, which corresponds to the color-magnitute $(\mathrm{CM})$ relation of elliptical galaxies in the present universe, for models with different masses $\left(M_{\mathrm{d}}=10^{10}, 10^{11}\right.$, and $\left.10^{12} M_{\odot}\right)$ and strength of supernova feedback effects $\left(f_{\mathrm{sn}}=0.0,0.1\right.$, and 0.5$)$. The results for models with $f_{\mathrm{sn}}=0.0$ (no feedback effects), 0.1 , and 0.5 are plotted by open squares, circles, and triangles, respectively. The results with larger $L_{B}$ mean those with larger $M_{\mathrm{d}}$. For clarity, the results for models with the same 
$f_{\text {sn }}$ are connected by a dotted line. For comparison, the observed $V-K$ color-magnitude relation (Bower et al. 1992) is given by a dash-dotted line in the bottom panel. Note that the CM relation can be more discernably observed in models with larger $f_{\mathrm{sn}}$.

Fig. 9.- The dependence of the central $\mathrm{Mg}_{2}$ index (within $0.1 R_{\mathrm{e}}$ ) on the central velocity dispersion $(\sigma)$ in merger remnants at the age of 13.1 Gyr for models with different masses $\left(M_{\mathrm{d}}=10^{10}, 10^{11}\right.$, and $\left.10^{12} M_{\odot}\right)$ and strength of supernova feedback effects $\left(f_{\mathrm{sn}}=0.0,0.1\right.$, and 0.5 ). The results for models with $f_{\mathrm{sn}}=0.0$ (no feedback effects), 0.1 , and 0.5 are plotted by open squares, circles, and triangles, respectively. The results with larger $\sigma$ mean those with larger $M_{\mathrm{d}}$. For clarity, the results for models with the same $f_{\mathrm{sn}}$ are connected by a dotted line. For comparison, the observed $\mathrm{Mg}_{2}-\sigma$ relation (Burstein et al. 1988) is given by a dash-dotted line. Note that the $\mathrm{Mg}_{2}-\sigma$ relation can be more discernably observed in models with larger $f_{\mathrm{sn}}$.

Fig. 10.- The luminosity $\left(L_{B}\right.$ and $L_{K}$ in units of $\left.L_{\odot}\right)$ dependence of mass-to-light-ratio $\left(M / L_{B}\right.$ and $\left.M / L_{K}\right)$ in merger remnants at the age of $13.1 \mathrm{Gyr}$ for models with different masses $\left(M_{\mathrm{d}}=10^{10}, 10^{11}\right.$, and $\left.10^{12} M_{\odot}\right)$ and strength of supernova feedback effects $\left(f_{\mathrm{sn}}=0.0\right.$, 0.1 , and 0.5 ). Here mas-to-light-ratio means the ratio of total stellar mass (not total mass of a merger remnant) to total stellar light in a merger remnant. The results for models with $f_{\mathrm{sn}}$ $=0.0$ (no feedback effects), 0.1, and 0.5 are plotted by open squares, circles, and triangles, respectively. The results with larger $L_{B}$ and $L_{K}$ mean those with larger $M_{\mathrm{d}}$. For clarity, the results for models with the same $f_{\mathrm{sn}}$ are connected by a dotted line. For comparison, the observed luminosity dependeces of $M / L_{B}\left(M / L_{B} \propto L_{B}{ }^{0.2}\right.$ derived by Bender et al. 1992) and $M / L_{K}\left(M / L_{K} \propto L_{K}^{0.17}\right.$ by Pahre et al. 1998) are also given by solid lines with arbitrary zero-points.

Fig. 11. - The dependence of $U-R$ color gradient on mean stellar metallicity $\left(\left\langle Z_{*}\right\rangle\right)$ in merger remnants for models with $R_{\text {chem }}=0.1$ and gaseous dissipation (Model 1 and 2, open 
circles), those with $R_{\text {chem }}=0.4$ and gaseous dissipation (Model 10 and 11, filled squares), and those with $R_{\text {chem }}=0.1$ and no gaseous dissipation (Model 12 and 13, filled circles). In this figure, the results for three models with $M_{\mathrm{d}}=10^{10} M_{\odot}$ (Model 1, 10, and 12) and those for three models with $M_{\mathrm{d}}=10^{12} M_{\odot}$ (Model 2, 11, and 13) are given. The results with larger $\left\langle Z_{*}\right\rangle$ denote those for models with $M_{\mathrm{d}}=10^{12} M_{\odot}$. For clarity, the results for models with the same $R_{\text {chem }}$ and degree of gaseous dissipation (for example, Model 1 and 2) are connected by a dotted line.

Fig. 12. - The dependence of the radial gradient of $\mathrm{Mg}_{2}$ index $\left(\Delta \mathrm{Mg}_{2} / \Delta \log R\right)$ on the central $\mathrm{Mg}_{2}$ index in merger remnants for all models in the present study. For comparison, the observed depdence of González \& Gorgas (1996), $\Delta \mathrm{Mg}_{2} / \Delta \log \mathrm{R}=0.026-0.28 \mathrm{Mg}_{2}$, is also given by a solid line.

Fig. 13. - The dependence of the radial gradient of stellar metallicity $\left(\Delta \log \left\langle Z_{*}\right\rangle / \Delta \log R\right)$ on that of mean epoch of star formation $\left(\Delta \log \left\langle T_{\mathrm{SF}}\right\rangle / \Delta \log R\right)$ in merger remnants for all models in the present study. Here merger remnants with larger value of $\left\langle T_{\mathrm{SF}}\right\rangle$ have a larger amount of younger stellar populations. Note that merger remnants with steeper age gradient show steeper metallcity gradient.

Fig. 14.- Time evolution of total mass in units of $M_{\odot}$ for metals Z $\left(m_{\mathrm{Z}}\right.$, top), $\mathrm{O}\left(m_{\mathrm{O}}\right.$, second from the top), $\mathrm{Fe}$ ( $m_{\mathrm{Fe}}$, second from the bottom), and $\mathrm{Mg}\left(m_{\mathrm{Mg}}\right.$, bottom), ejected from stars with variously different masses and lifetimes in a SSP with solar metallicity and solar mass $\left(1.0 M_{\odot}\right)$ for the following three models: Model A with the Salpeter IMF, $M_{\text {low }}=0.1 M_{\odot}$, and $M_{\text {up }}=120.0 M_{\odot}$ (solid lines), Model B with the Salpeter IMF, $M_{\text {low }}=0.6 M_{\odot}$, and $M_{\text {up }}=120.0 M_{\odot}$ (dotted lines), and Model C with IMF with shallower slope (the exponent of IMF equal to -1.1), $M_{\text {low }}=0.1 M_{\odot}$, and $M_{\text {up }}=120.0 M_{\odot}$ (dashed lines). The time evolution of Model B corresponds to $Y_{i, j}(t)$ adopted in the main manuscript. 
Fig. 15. - Long-term evolution of the abundance ratio of $[\mathrm{Mg} / \mathrm{Fe}]$ in a one-zone chemical evolution model of a disk galaxy for Model A (solid line), B (dotted one), and C (dashed one). Details of the one-zone disk model are given in the manuscript.

Fig. 16. - Time evolution of $M / L_{B}, B-V$, and $V-K$ color in a SSP with solar metallicity for Model A (solid lines), B (dotted ones), and C (dashed ones). Here $M / L_{B}$ means the ratio of stellar mass to $B$-band luminosity. Note that $M / L_{B}$ depends more strongly on IMF and $M_{\text {low }}$ whereas $B-V$ and $V-K$ color do not. 